\title{
Option Pricing and Sensitivity Analysis in the Lévy Forward Process Model
}

\author{
Ernst Eberlein, M'hamed Eddahbi and Sidi Mohamed Lalaoui Ben Cherif
}

\begin{abstract}
The purpose of this article is to give a closed Fourier-based valuation formula for a caplet in the framework of the Lévy forward process model which was introduced in Eberlein and Özkan, Financ. Stochast. 9:327-348, 2005, [5]. Afterwards, we compute Greeks by two approaches which come from totally different mathematical fields. The first is based on the integration-by-parts formula, which lies at the core of the application of the Malliavin calculus to finance. The second consists in using Fourier-based methods for pricing derivatives as exposed in Eberlein, Quantitative Energy Finance, 2014, [3]. We illustrate the results in the case where the jump part of the underlying model is driven by a time-inhomogeneous Gamma process and alternatively by a Variance Gamma process.
\end{abstract}

Keywords Option valuation - Lévy forward process model • Fourier transform • Time-inhomogeneous Lévy processes $\cdot$ Malliavin calculus $\cdot$ Greeks and sensitivity analysis

We acknowledge financial support from the Federal Foreign Office of Germany which has been granted within a program of the German Academic Exchange Service (DAAD).

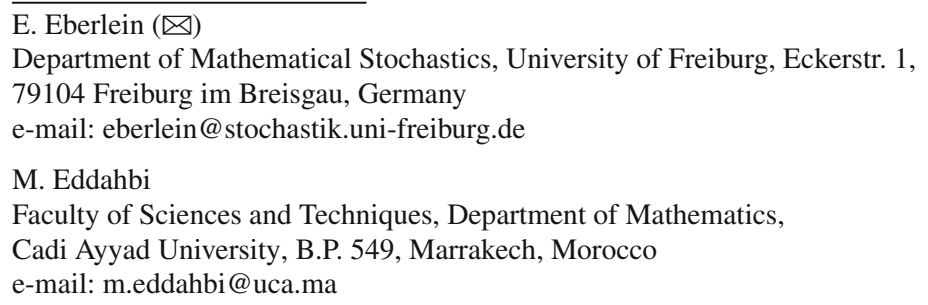

S.M. Lalaoui Ben Cherif

Faculty of Sciences Semlalia, Department of Mathematics, Cadi Ayyad University,

B.P. 2390, Marrakech, Morocco

e-mail:mohamed.lalaoui@ced.uca.ac.ma 


\section{Introduction}

To compute expectations which arise as prices of derivative products is a key issue in quantitative finance. The effort which is necessary to get these values depends to a high degree on the sophistication of the model approach which is used. Simple models such as the classical geometric Brownian motion lead to easy-to-evaluate formulas for expectations but entail at the same time a high model risk. As has been shown in numerous studies, the empirical return distributions which one can observe are far from normality. This is true for all categories of financial markets: equity, fixed income, foreign exchange as well as credit markets (see e.g. Eberlein and Keller (1995) [4] for the analysis of stock price data and Eberlein and Kluge (2007) [7] for data from fixed income markets). A first step to reduce model risk and to improve the performance of the model consists in introducing volatility as a stochastic quantity. Some of the stochastic volatility models became quite popular. Nevertheless one must be aware that the distributions which diffusion processes with non-deterministic coefficients generate on a given time horizon are not known. They can only be determined approximately on the basis of simulations of process paths. In order to get more realistic distributions, an excellent choice is to replace the driving Brownian motion in classical models by a suitably chosen Lévy process. This can also be interpreted in the sense that instead of making volatility stochastic one can go over to a stochastic clock. The reason is that many Lévy processes can be obtained as time-changed Brownian motions. For example, the Variance Gamma process results when one replaces linear time by a Gamma process as subordinator. Of course, one can also consider both: a more powerful driver and stochastic volatility.

Lévy processes are in a one-to-one correspondence to the rich class of infinitely divisible distributions and at the same time analytically well tractable. Due to the higher number of available parameters, this class of distributions is flexible enough to allow a much better fit to empirical return distributions. The systematic error which results from the assumption of normality is avoided. The generating distribution of a Lévy process shows up as the distribution of increments of length one. Consequently, any distribution which one gets by fitting a parametrized subclass to empirical return data can be implemented not only approximately but exactly into Lévy-driven models. Suitably parametrized model classes which have been used successfully so far are driven by generalized hyperbolic, normal inverse Gaussian (NIG), or Variance Gamma (VG) processes, just to mention a few.

As noted above, advanced models with superior statistical properties require more demanding numerical methods. Efficient and accurate algorithms are crucial in this context, in particular for calibration purposes. For pricing of derivatives the historical distribution, which can be derived from price data of the underlying and which is used for risk management, is of less interest. Calibration usually means to estimate the risk-neutral distribution parameters. In other words, one exploits price data of derivatives. In most cases this is given in terms of volatilities. Whereas years ago calibration was usually done overnight, many trading desks recalibrate nowadays on an intraday basis. During a calibration procedure in each iteration step a large 
number of model prices have to be computed and compared to market prices. A method which almost always works to get the corresponding expectations is Monte Carlo simulation. Its disadvantage is that it is computationally intensive and therefore too slow for many purposes. Another classical approach is to represent prices as solutions of partial differential equations (PDEs) which in the case of Lévy processes with jumps become partial integro-differential equations (PIDEs). This approach, which is based on the Feynman-Kac formula, applies to a wide range of valuation problems, in particular it allows to compute prices of American options as well. Nevertheless, the numerical solution of PIDEs rests on sophisticated discretization methods and corresponding programs. In this paper we concentrate on the third, namely the Fourier-based approach.

To manage portfolios of derivatives, traders have to understand how sensitive prices of derivative products are with respect to changes in the underlying parameters. For this purpose they need to know the Greeks which are given by the partial derivatives of the pricing functional with respect to those parameters. Usually Greeks are estimated by means of a finite difference approximation. Two kinds of errors are produced this way: the first one comes from the approximation of the derivative by a finite difference and the second one results from the numerical computation of the expectation. To eliminate one of the sources of error, Fournié et al. (1999) [9] adopted a new approach which consists in shifting the differential operator from the pricing functional to the diffusion kernel. This procedure results in an expectation operator applied to the payoff multiplied by a random weight function.

In the following we focus on a discrete tenor interest rate model which has been introduced in Eberlein and Özkan (2005) [5]. This so-called Lévy forward process model is driven by a time-inhomogeneous Lévy process and is developed on the basis of a backward induction that is necessary to get the LIBOR rates in a convenient homogeneous form. A major advantage of the forward process approach is that it is invariant under the measure change in the sense that the driving process remains a time-inhomogeneous Lévy process. Moreover, the measure changes do not only have the invariance property but in addition they are analytically and consequently also numerically much simpler compared to the corresponding measure changes in the so-called LIBOR model. The reason is that in each induction step the forward process itself represents up to a norming constant the density process on which the measure change is based. As a consequence, any approximation such as the 'frozen drift' approximation or more sophisticated versions of it are completely avoided. This means that the approximation error with which one has to struggle in the LIBOR approach does not show up in the forward process approach.

Another important aspect is that in the latter model the increments of the driving process translate directly into increments of the LIBOR rates. This is not the case for the LIBOR model where the increments of the LIBOR rates are proportional to the corresponding increments of the driving process scaled with the current value of the LIBOR rate. Expressed in terms of the terminology which will be developed in Sects. 2 and 3 this means that in the Lévy LIBOR model 


$$
L\left(t+\Delta t, T_{k}\right)-L\left(t, T_{k}\right) \sim L\left(t, T_{k}\right)\left(L_{t+\Delta t}^{T_{k+1}}-L_{t}^{T_{k+1}}\right)
$$

whereas in the Lévy forward process model

$$
L\left(t+\Delta t, T_{k}\right)-L\left(t, T_{k}\right) \sim \delta_{k}^{-1}\left(L_{t+\Delta t}^{T_{k+1}}-L_{t}^{T_{k+1}}\right) .
$$

The fact that the increments of the LIBOR rate process do not depend on current LIBOR values, translates into increased flexibility and a superior model performance of the forward process approach.

In addition to the differences in mathematical properties there is a fundamental economic difference. The forward process approach allows for negative interest rates as well as for negative starting values. This is of crucial importance in particular in the current economic environment where negative rates are common. Models where by construction interest rates stay strictly positive are not able to produce realistic valuations for a large collection of interest rate derivatives in a deflationary or neardeflationary environment.

As far as the calculation of Greeks in this setting is concerned, we refer to Glasserman and Zhao (1999) [12], Glasserman (2004) [11], and Fries (2007) [10] where some treatment of this issue is given. The classical diffusion-based LIBOR market model offers a high degree of analytical tractability. However, this model cannot reproduce the phenomenon of changing volatility smiles along the maturity axis. In order to gain more flexibility in a first step one can replace the driving Brownian motion by a (time-homogeneous) Lévy process. However, one observes that the shape of the volatility surface produced by cap and floor prices is too sophisticated in order to be matched with sufficient accuracy by a model which is driven by a time-homogeneous process. To achieve a more accurate calibration of the model across different strikes and maturities one has to use the more flexible class of time-inhomogeneous Lévy processes (see e.g. Eberlein and Özkan (2005) [5] and Eberlein and Kluge (2006) [6]). Graphs in the latter paper show in particular that interest rate models driven by time-inhomogeneous Lévy processes are able to reproduce implied volatility curves (smiles) observed in the market across all maturities with high accuracy. If one restricts the approach to (time-homogeneous) Lévy processes as drivers, the smiles flatten out too fast at longer maturities. Consequently, we have analyticalthe invariance under measure changes - as well as statistical reasons to choose timeinhomogeneous Lévy processes as drivers. In implementations of the model already a rather mild form of time-inhomogeneity turns out to be sufficient. Typically one has to glue together three pieces of (time-homogeneous) Lévy processes in order to cover the full range of maturities with sufficient accuracy. In terms of parameters this means that instead of three or four one uses nine or twelve parameters.

The first goal of this paper is to give a closed Fourier-based valuation formula for a caplet in the framework of the Lévy forward process model. The second aim is to study sensitivities. We discuss two approaches for this purpose. The first is based on the integration-by-parts formula, which lies at the core of the application of the Malliavin calculus to finance as developed in Fournié et al. (1999) [9], León et al. 
(2002) [14], Petrou (2008) [17], Yablonski (2008) [19]. This approach is appropriate if the driving process has a diffusion component. The second approach which covers purely discontinuous drivers as well relies on Fourier-based methods for pricing derivatives. For a survey of Fourier-based methods see Eberlein (2014) [3]. We illustrate the result by applying the formula to the pricing of a caplet where the jumppart of the underlying model is driven by a time-inhomogeneous Gamma process and alternatively by a Variance Gamma process.

\section{The Lévy Forward Process Model}

Let $0=T_{0}<T_{1}<\cdots<T_{n-1}<T_{n}=T^{*}$ denote a discrete tenor structure and set $\delta_{k}=T_{k+1}-T_{k}$ for all $k \in\{0, \ldots, n-1\}$. Because we proceed by backward induction, let us use the notation $T_{i}^{*}:=T_{n-i}$ and $\delta_{i}^{*}=\delta_{n-i}$ for $i \in\{1, \ldots, n\}$. For zerocoupon bond prices $B\left(t, T_{i}^{*}\right)$ and $B\left(t, T_{i-1}^{*}\right)$, the forward process is defined by

$$
F\left(t, T_{i}^{*}, T_{i-1}^{*}\right)=\frac{B\left(t, T_{i}^{*}\right)}{B\left(t, T_{i-1}^{*}\right)} .
$$

Hence, modeling forward processes means specifying the dynamics of ratios of successive bond prices. Let $\left(\Omega ; \mathscr{F}=\mathscr{F}_{T^{*}} ; \mathbb{F} ; \mathbb{P}_{T^{*}}\right)$ be a complete stochastic basis where $\mathbb{P}_{T^{*}}$ should be regarded as the forward martingale measure for the settlement date $T^{*}>0$ and the filtration $\mathbb{F}=\left(\mathscr{F}_{t}\right)_{t \in\left[0, T^{*}\right]}$ satisfies the usual conditions. Consider a time-inhomogeneous Lévy process $L^{T^{*}}$ defined on $\left(\Omega ; \mathscr{F}=\mathscr{F}_{T^{*}} ; \mathbb{F} ; \mathbb{P}_{T^{*}}\right)$ starting at 0 with local characteristics $\left(b^{T^{*}}, c, F^{T^{*}}\right)$ such that the drift term $b_{s}^{T^{*}} \in \mathbb{R}$, the volatility coefficient $c_{s}$ and the Lévy measure $F_{s}^{T^{*}}$ satisfy the following conditions

$$
\exists \sigma>0, \quad \forall s \in\left[0, T^{*}\right]: c_{s}>\sigma, F_{s}^{T^{*}}(\{0\})=0
$$

and

$$
\int_{0}^{T^{*}}\left(\left|b_{s}^{T^{*}}\right|+\left|c_{s}\right|+\int_{\mathbb{R}}\left(|x|^{2} \wedge 1\right) F_{s}^{T^{*}}(d x)\right) d s<\infty .
$$

We impose as usual a further integrability condition. Note that the processes which we will define later, are by construction martingales and therefore every single random variable has to be integrable.

Assumption 2.1 (EM) There exists a constant $M>1$ such that

$$
\int_{0}^{T^{*}} \int_{\{|x|>1\}} \exp (u x) F_{s}^{T^{*}}(d x) d s<\infty, \quad \forall u \in[-M, M] .
$$


Under $(\mathbb{E M})$ the random variable $L_{t}^{T^{*}}$ has a finite expectation and its law is given by the characteristic function

$$
\mathbb{E}\left[e^{\mathrm{i} u L_{t}^{T^{*}}}\right]=\exp \left(\int_{0}^{t}\left(\mathrm{i} u b_{s}^{T^{*}}-\frac{1}{2} u^{2} c_{s}+\int_{\mathbb{R}}\left(e^{\mathrm{i} u x}-1-\mathrm{i} u x\right) F_{s}^{T^{*}}(d x)\right) d s\right) .
$$

Furthermore, the process $L^{T^{*}}$ is a special semimartingale, and thus its canonical representation has the simple form

$$
L_{t}^{T^{*}}=\int_{0}^{t} b_{s}^{T^{*}} d s+\int_{0}^{t} \sqrt{c_{s}} d W_{s}^{T^{*}}+\int_{0}^{t} \int_{\mathbb{R}} x \widetilde{\mu}^{L^{T^{*}}}(d s, d x)
$$

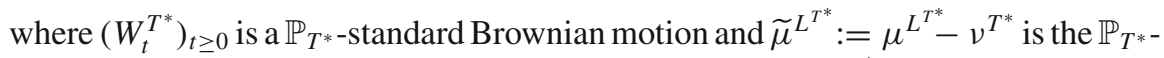
compensated random measure of jumps of $L^{T^{*}}$. As usual, $\mu^{L^{T^{*}}}$ denotes the random measure of jumps of $L^{T^{*}}$ and $v^{T^{*}}(d s, d x):=F_{s}^{T^{*}}(d x) d s$ the $\mathbb{P}_{T^{*} \text {-compensator of }}$ $\mu^{L^{T^{*}}}$. We denote by $\theta_{s}$ the cumulant function associated with the process $L^{T^{*}}$ as given in (8) with local characteristics $\left(b^{T^{*}}, c, F^{T^{*}}\right)$, that is, for appropriate $z \in \mathbb{C}$

$$
\theta_{s}(z)=z b_{s}^{T^{*}}+\frac{z^{2}}{2} c_{s}+\int_{\mathbb{R}}\left(e^{z x}-1-z x\right) F_{s}^{T^{*}}(d x),
$$

where $c$ and $F^{T^{*}}$ are free parameters, whereas the drift characteristic $b^{T^{*}}$ will later be chosen to guarantee that the forward process is a martingale. The following ingredients are needed.

Assumption 2.2 ( $\mathbb{L} \mathbb{R} .1)$ For any maturity $T_{i}^{*}$ there is a bounded, deterministic function $\lambda\left(\cdot, T_{i}^{*}\right):\left[0, T^{*}\right] \longmapsto \mathbb{R}$ which represents the volatility of the forward process $F\left(\cdot, T_{i}^{*}, T_{i-1}^{*}\right)$. These functions satisfy

$\lambda\left(s, T_{i}^{*}\right)>0, \forall s \in\left[0, T_{i}^{*}\right]$ and $\lambda\left(s, T_{i}^{*}\right)=0$ for $s>T_{i}^{*}$ for any maturity $T_{i}^{*}$, $\sum_{i=1}^{n-1} \lambda\left(s, T_{i}^{*}\right) \leq M, \forall s \in\left[0, T^{*}\right]$ where $M$ is the constant from Assumption $(\mathbb{E M})$.

Assumption $2.3(\mathbb{L} \mathbb{R} .2)$ The initial term structure of zero-coupon bond prices $B\left(0, T_{i}^{*}\right)$ is strictly positive for all $i \in\{1, \ldots, n\}$.

We begin to construct the forward process with the most distant maturity and postulate

$$
F\left(t, T_{1}^{*}, T^{*}\right)=F\left(0, T_{1}^{*}, T^{*}\right) \exp \left(\int_{0}^{t} \lambda\left(s, T_{1}^{*}\right) d L_{s}^{T^{*}}\right)
$$

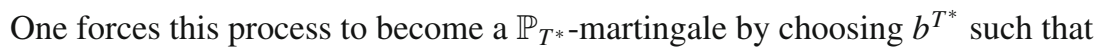




$$
\begin{aligned}
\int_{0}^{t} \lambda\left(s, T_{1}^{*}\right) b_{s}^{T^{*}} d s= & -\frac{1}{2} \int_{0}^{t} c_{s} \lambda^{2}\left(s, T_{1}^{*}\right) d s \\
& -\int_{0}^{t} \int_{\mathbb{R}}\left(e^{x \lambda\left(s, T_{1}^{*}\right)}-1-x \lambda\left(s, T_{1}^{*}\right)\right) v^{T^{*}}(d s, d x) .
\end{aligned}
$$

Then the forward process $F\left(\cdot, T_{1}^{*}, T^{*}\right)$ can be given as a stochastic exponential

$$
F\left(t, T_{1}^{*}, T^{*}\right)=F\left(0, T_{1}^{*}, T^{*}\right) \mathscr{E}_{t}\left(Z\left(\cdot, T_{1}^{*}\right)\right)
$$

with

$$
Z\left(t, T_{1}^{*}\right)=\int_{0}^{t} \sqrt{c_{s}} \lambda\left(s, T_{1}^{*}\right) d W_{s}^{T^{*}}+\int_{0}^{t} \int_{\mathbb{R}}\left(e^{x \lambda\left(s, T_{1}^{*}\right)}-1\right) \widetilde{\mu}^{L^{T^{*}}}(d s, d x) .
$$

Since the forward process $F\left(\cdot, T_{1}^{*}, T^{*}\right)$ is a $\mathbb{P}_{T^{*}-\text { martingale, we can use it as a density }}$ process and define the forward martingale measure $\mathbb{P}_{T_{1}^{*}}$ by setting

$$
\frac{d \mathbb{P}_{T_{1}^{*}}}{d \mathbb{P}_{T^{*}}}=\frac{F\left(T_{1}^{*}, T_{1}^{*}, T^{*}\right)}{F\left(0, T_{1}^{*}, T^{*}\right)}=\mathscr{E}_{T_{1}^{*}}\left(Z\left(\cdot, T_{1}^{*}\right)\right) .
$$

By the semimartingale version of Girsanov's theorem (see Jacod and Shiryaev (1987) [13])

$$
W_{t}^{T_{1}^{*}}:=W_{t}^{T^{*}}-\int_{0}^{t} \sqrt{c_{s}} \lambda\left(s, T_{1}^{*}\right) d s
$$

is a $\mathbb{P}_{T_{1}^{*} \text {-standard Brownian motion and }}$

$$
v^{T_{1}^{*}}(d t, d x):=e^{x \lambda\left(s, T_{1}^{*}\right)} v^{T^{*}}(d t, d x)=e^{x \lambda\left(s, T_{1}^{*}\right)} F_{s}^{T^{*}}(d x) d s
$$

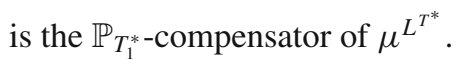

Continuing this way one gets the forward processes $F\left(\cdot, T_{i}^{*}, T_{i-1}^{*}\right)$ such that for all $i \in\{1, \ldots, n\}$

$$
F\left(t, T_{i}^{*}, T_{i-1}^{*}\right)=F\left(0, T_{i}^{*}, T_{i-1}^{*}\right) \exp \left(\int_{0}^{t} \lambda\left(s, T_{i}^{*}\right) d L_{s}^{T_{i-1}^{*}}\right) .
$$

The drift term $b^{T_{i-1}^{*}}$ is chosen in such a way that the forward process $F\left(\cdot, T_{i}^{*}, T_{i-1}^{*}\right)$ becomes a martingale under the forward measure $\mathbb{P}_{T_{i-1}^{*}}$, that is

$$
\begin{aligned}
\int_{0}^{t} \lambda\left(s, T_{i}^{*}\right) b_{s}^{T_{i-1}^{*}} d s= & -\frac{1}{2} \int_{0}^{t} c_{s} \lambda^{2}\left(s, T_{i}^{*}\right) d s \\
& -\int_{0}^{t} \int_{\mathbb{R}}\left(e^{x \lambda\left(s, T_{i}^{*}\right)}-1-x \lambda\left(s, T_{i}^{*}\right)\right) v^{T_{i-1}^{*}}(d s, d x) .
\end{aligned}
$$


We propose the following choice for the functions $b^{T_{i-1}^{*}}$ for all $i \in\{1, \ldots, n\}$

$$
\left\{\begin{array}{cc}
b_{s}^{T_{i-1}^{*}}=-\frac{c_{s}}{2} \lambda\left(s, T_{i}^{*}\right)-\int_{\mathbb{R}}\left(\frac{e^{x \lambda\left(s, T_{i}^{*}\right)}-1}{\lambda\left(s, T_{i}^{*}\right)}-x\right) F_{s}^{T_{i-1}^{*}}(d x), 0 \leq s<T_{i}^{*} \\
b_{s}^{T_{i-1}^{*}}=0, & s \geq T_{i}^{*} .
\end{array}\right.
$$

The driving process $L^{T_{i-1}^{*}}$ becomes therefore

$$
\begin{aligned}
L_{t}^{T_{i-1}^{*}}= & -\int_{0}^{t}\left(\frac{c_{s}}{2} \lambda\left(s, T_{i}^{*}\right)+\int_{\mathbb{R}}\left(\frac{e^{x \lambda\left(s, T_{i}^{*}\right)}-1}{\lambda\left(s, T_{i}^{*}\right)}-x\right) F_{s}^{T_{i-1}^{*}}(d x)\right) d s \\
& +\int_{0}^{t} \sqrt{c_{s}} d W_{s}^{T_{i-1}^{*}}+\int_{0}^{t} \int_{\mathbb{R}} x\left(\mu^{T^{*}}-v^{T_{i-1}^{*}}\right)(d s, d x)
\end{aligned}
$$

under the successive forward measures $\mathbb{P}_{T_{i}^{*}}$ which are given by the recursive relation

$$
\left\{\begin{aligned}
\frac{d \mathbb{P}_{T_{i}^{*}}}{d \mathbb{P}_{T_{i-1}^{*}}} & =\frac{F\left(T_{i}^{*}, T_{i}^{*}, T_{i-1}^{*}\right)}{F\left(0, T_{i}^{*}, T_{i-1}^{*}\right)}=\mathscr{E}_{T_{i}^{*}}\left(Z\left(\cdot, T_{i}^{*}\right)\right), \quad i \in\{1, \ldots, n\} \\
\mathbb{P}_{T_{0}^{*}} & =\mathbb{P}_{T^{*}}
\end{aligned}\right.
$$

with

$$
Z\left(t, T_{i}^{*}\right)=\int_{0}^{t} \sqrt{c_{s}} \lambda\left(s, T_{i}^{*}\right) d W_{s}^{T_{i-1}^{*}}+\int_{0}^{t} \int_{\mathbb{R}}\left(e^{x \lambda\left(s, T_{i}^{*}\right)}-1\right) \widetilde{\mu}^{L^{T_{i-1}^{*}}}(d s, d x),
$$

where $\left(W_{t}^{T_{i-1}^{*}}\right)_{t \geq 0}$ is a $\mathbb{P}_{T_{i-1}^{*}}$-standard Brownian motion such that

$$
\left\{\begin{array}{l}
W_{t}^{T_{i}^{*}}=W_{t}^{T_{i-1}^{*}}-\int_{0}^{t} \sqrt{c_{s}} \lambda\left(s, T_{i}^{*}\right) d s, \quad i \in\{1, \ldots, n\} \\
W_{t}^{T_{0}^{*}}=W_{t}^{T^{*}}
\end{array}\right.
$$

$\widetilde{\mu}_{i-1}^{L^{T^{*}}}:=\mu^{L^{T^{*}}}-v^{T_{i-1}^{*}}$ is the $\mathbb{P}_{T_{i-1}^{*}}$-compensated random measure of jumps of $L^{T^{*}}$ and $v^{T_{i-1}^{*}}(d s, d x)=F_{s}^{T_{i-1}^{*}}(d x) d s$ is the $\mathbb{P}_{T_{i-1}^{*}}$-compensator of $\mu^{L^{T^{*}}}$ such that

$$
\left\{\begin{array}{l}
F_{s}^{T_{i}^{*}}(d x)=e^{x \lambda\left(s, T_{i}^{*}\right)} F_{s}^{T_{i-1}^{*}}(d x), \quad i \in\{1, \ldots, n\} \\
F_{s}^{T_{0}^{*}}(d x)=F_{s}^{T^{*}}(d x)
\end{array}\right.
$$


Setting $\Lambda^{i}(s):=\sum_{j=1}^{i} \lambda\left(s, T_{j}^{*}\right)$, we conclude that for all $i \in\{1, \ldots, n\}$

$$
W_{t}^{T_{i}^{*}}=W_{t}^{T^{*}}-\int_{0}^{t} \sqrt{c_{s}} \Lambda^{i}(s) d s
$$

and

$$
F_{s}^{T_{i}^{*}}(d x)=\exp \left(x \Lambda^{i}(s)\right) F_{s}^{T^{*}}(d x) .
$$

Note that the coefficients $\sqrt{c_{s}} \Lambda^{i}(s)$ and $\exp \left(x \Lambda^{i}(s)\right)$, which appear in this measure change, are deterministic functions and therefore the measure change is structure preserving, i.e. the driving process is still a time-inhomogeneous Lévy process after the measure change.

Since the forward process $F\left(\cdot, T_{i}^{*}, T_{i-1}^{*}\right)$ is by construction a $\mathbb{P}_{T_{i-1}^{*}}$-martingale, the process $\frac{F\left(\cdot, T_{i}^{*}, T_{i-1}^{*}\right)}{F\left(0, T_{i}^{*}, T_{i-1}^{*}\right)}$, which is the density process

$$
\left.\frac{d \mathbb{P}_{T_{i}^{*}}}{d \mathbb{P}_{T_{i-1}^{*}}^{*}}\right|_{\mathscr{F}_{t}}=\frac{F\left(t, T_{i}^{*}, T_{i-1}^{*}\right)}{F\left(0, T_{i}^{*}, T_{i-1}^{*}\right)}
$$

is a $\mathbb{P}_{T_{i-1}^{*}}$-martingale as well. By iterating the relation (21) we get on $\mathscr{F}_{T_{i-1}^{*}}$

$$
\begin{aligned}
\frac{d \mathbb{P}_{T_{i-1}^{*}}}{d \mathbb{P}_{T^{*}}} & =\frac{B\left(0, T^{*}\right)}{B\left(0, T_{i-1}^{*}\right)} \prod_{j=1}^{i-1} F\left(T_{i-1}^{*}, T_{j}^{*}, T_{j-1}^{*}\right) \\
& =\exp \left(\sum_{j=1}^{i-1} \int_{0}^{T_{i-1}^{*}} \lambda\left(s, T_{j}^{*}\right) d L_{s}^{T_{j-1}^{*}}\right) .
\end{aligned}
$$

Applying Proposition III.3.8 of Jacod and Shiryaev (1987) [13], we see that its restriction to $\mathscr{F}_{t}$ for $t \in\left[0, T_{i}^{*}\right]$

$$
\left.\frac{d \mathbb{P}_{T_{i}^{*}}}{d \mathbb{P}_{T^{*}}}\right|_{\mathscr{F}_{t}}=\frac{B\left(0, T^{*}\right)}{B\left(0, T_{i}^{*}\right)} \prod_{j=1}^{i} F\left(t, T_{j}^{*}, T_{j-1}^{*}\right)
$$

is a $\mathbb{P}_{T^{*}-\text { martingale. }}$

\section{Fourier-Based Methods for Option Pricing}

We will derive an explicit valuation formula for standard interest rate derivatives such as caps and floors in the Lévy forward process model. Since floor prices can 
be derived from the corresponding put-call-parity relation we concentrate on caps. Recall that a cap is a sequence of call options on subsequent LIBOR rates. Each single option is called a caplet. The payoff of a caplet with strike rate $K$ and maturity $T_{i}^{*}$ is

$$
\delta_{i}^{*}\left(L\left(T_{i}^{*}, T_{i}^{*}\right)-K\right)^{+}
$$

where the payment is made at time point $T_{i-1}^{*}$. The forward LIBOR rates $L\left(T_{i}^{*}, T_{i}^{*}\right)$ are the discretely compounded, annualized interest rates which can be earned from investment during a future interval starting at $T_{i}^{*}$ and ending at $T_{i-1}^{*}$ considered at the time point $T_{i}^{*}$. These rates can be expressed in terms of the forward prices as follows

$$
L\left(T_{i}^{*}, T_{i}^{*}\right)=\frac{1}{\delta_{i}^{*}}\left(F\left(T_{i}^{*}, T_{i}^{*}, T_{i-1}^{*}\right)-1\right)
$$

Its time-0-price, denoted by $\operatorname{Cplt}_{0}\left(T_{i}^{*}, K\right)$, is given by

$$
\operatorname{Cplt}_{0}\left(T_{i}^{*}, K\right)=B\left(0, T_{i-1}^{*}\right) \delta_{i}^{*} \mathbb{E}_{\mathbb{P}_{T_{i-1}^{*}}}\left[\left(L\left(T_{i}^{*}, T_{i}^{*}\right)-K\right)^{+}\right] .
$$

Instead of basing the pricing on the Lévy LIBOR model one can use the Lévy forward process approach (see Eberlein and Özkan (2005) [5]). It is then more natural to write the pricing formula (32) in the form

$$
\operatorname{Cplt}_{0}\left(T_{i}^{*}, K\right)=B\left(0, T_{i-1}^{*}\right) \mathbb{E}_{\mathbb{P}_{T_{i-1}^{*}}}\left[\left(F\left(T_{i}^{*}, T_{i}^{*}, T_{i-1}^{*}\right)-\widetilde{K}_{i}\right)^{+}\right]
$$

where $\widetilde{K}_{i}:=1+\delta_{i}^{*} K$. From (17), the forward process $F\left(\cdot, T_{i}^{*}, T_{i-1}^{*}\right)$ is given by

$$
\begin{aligned}
F\left(T_{i}^{*}, T_{i}^{*}, T_{i-1}^{*}\right)= & F\left(0, T_{i}^{*}, T_{i-1}^{*}\right) \exp \left(\int_{0}^{T_{i}^{*}} b_{s}^{T_{i-1}^{*}} \lambda\left(s, T_{i}^{*}\right) d s\right) \\
& \times \exp \left(\int_{0}^{T_{i}^{*}} \sqrt{c_{s}} \lambda\left(s, T_{i}^{*}\right) d W_{s}^{T_{i-1}^{*}}\right) \\
& \times \exp \left(\int_{0}^{T_{i}^{*}} \int_{\mathbb{R}} x \lambda\left(s, T_{i}^{*}\right) \widetilde{\mu}_{i-1}^{L^{T^{*}}}(d s, d x)\right) .
\end{aligned}
$$

Using the relations (25) and (26) we obtain for $t \in\left[0, T_{i}^{*}\right]$

$$
F\left(t, T_{i}^{*}, T_{i-1}^{*}\right)=F\left(0, T_{i}^{*}, T_{i-1}^{*}\right) \exp \left(\int_{0}^{t} \lambda\left(s, T_{i}^{*}\right) d L_{s}^{T^{*}}+d\left(t, T_{i}^{*}\right)\right)
$$


where

$$
\begin{aligned}
d\left(t, T_{i}^{*}\right)= & \int_{0}^{t} \lambda\left(s, T_{i}^{*}\right)\left[b_{s}^{T_{i-1}^{*}}-b_{s}^{T^{*}}-\Lambda^{i-1}(s) c_{s}\right] d s \\
& -\int_{0}^{t} \lambda\left(s, T_{i}^{*}\right) \int_{\mathbb{R}} x\left(e^{x \Lambda^{i-1}(s)}-1\right) F_{s}^{T^{*}}(d x) d s .
\end{aligned}
$$

Remember that on $\mathscr{F}_{T_{i-1}^{*}}$

$$
\frac{d \mathbb{P}_{T_{i-1}^{*}}}{d \mathbb{P}_{T^{*}}}=\exp \left(\sum_{j=1}^{i-1} \int_{0}^{T_{i-1}^{*}} \lambda\left(s, T_{j}^{*}\right) d L_{s}^{T^{*}}+\sum_{j=1}^{i-1} d\left(T_{i-1}^{*}, T_{j}^{*}\right)\right) .
$$

Keeping in mind Assumption $2.2(\mathbb{L} \mathbb{R} .1)$, we find

$$
\exp \left(-\sum_{j=1}^{i-1} d\left(T_{i-1}^{*}, T_{j}^{*}\right)\right)=\mathbb{E}_{\mathbb{P}_{T^{*}}}\left[\exp \left(\int_{0}^{T_{i-1}^{*}} \Lambda^{i-1}(s) d L_{s}^{T^{*}}\right)\right]
$$

Using Proposition 8 in Eberlein and Kluge (2006) [6], we find

$$
\exp \left(-\sum_{j=1}^{i-1} d\left(T_{i-1}^{*}, T_{j}^{*}\right)\right)=\exp \left(\int_{0}^{T_{i-1}^{*}} \theta_{s}\left(\Lambda^{i-1}(s)\right) d s\right) .
$$

Consequently,

$$
\frac{d \mathbb{P}_{T_{i-1}^{*}}}{d \mathbb{P}_{T^{*}}}=\exp \left(\int_{0}^{T_{i-1}^{*}} \Lambda^{i-1}(s) d L_{s}^{T^{*}}-\int_{0}^{T_{i-1}^{*}} \theta_{s}\left(\Lambda^{i-1}(s)\right) d s\right) .
$$

Knowing that the process $\left(\frac{F\left(\cdot, T_{i}^{*}, T_{i-1}^{*}\right)}{F\left(0, T_{i}^{*}, T_{i-1}^{*}\right)}\right)$ is a $\mathbb{P}_{T_{i-1}^{*}}$-martingale, we reach

$$
\exp \left(-d\left(T_{i}^{*}, T_{i}^{*}\right)\right)=\mathbb{E}_{\mathbb{P}_{T_{i-1}^{*}}}\left[\exp \left(\int_{0}^{T_{i}^{*}} \lambda\left(s, T_{i}^{*}\right) d L_{s}^{T^{*}}\right)\right]
$$

Hence,

$$
\begin{aligned}
& \exp \left(-d\left(T_{i}^{*}, T_{i}^{*}\right)\right) \\
& =\exp \left(-\int_{0}^{T_{i}^{*}} \theta_{s}\left(\Lambda^{i-1}(s)\right) d s\right) \mathbb{E}_{\mathbb{P}_{T^{*}}}\left[\exp \left(\int_{0}^{T_{i}^{*}} \Lambda^{i}(s) d L_{s}^{T^{*}}\right)\right] \\
& =\exp \left(\int_{0}^{T_{i}^{*}}\left[\theta_{s}\left(\Lambda^{i}(s)\right)-\theta_{s}\left(\Lambda^{i-1}(s)\right)\right] d s\right) .
\end{aligned}
$$


Thus,

$$
d\left(T_{i}^{*}, T_{i}^{*}\right)=\int_{0}^{T_{i}^{*}}\left[-\theta_{s}\left(\Lambda^{i}(s)\right)+\theta_{s}\left(\Lambda^{i-1}(s)\right)\right] d s .
$$

Define the random variable $X_{T_{i}^{*}}$ as the logarithm of $F\left(T_{i}^{*}, T_{i}^{*}, T_{i-1}^{*}\right)$. Therefore,

$$
X_{T_{i}^{*}}=\ln \left(F\left(0, T_{i}^{*}, T_{i-1}^{*}\right)\right)+\int_{0}^{T_{i}^{*}} \lambda\left(s, T_{i}^{*}\right) d L_{s}^{T^{*}}+d\left(T_{i}^{*}, T_{i}^{*}\right) .
$$

Proposition 3.1 Suppose there is a real number $R \in(1,1+\varepsilon)$ such that the moment-generating function of $X_{T_{i}^{*}}$ with respect to $\mathbb{P}_{T_{i-1}^{*}}$ is finite at $R$, i.e. $M_{X_{T_{i}^{*}}}(R)$ $<\infty$, then

$$
\begin{aligned}
& \operatorname{Cplt}_{0}\left(T_{i}^{*}, K\right)=\frac{\widetilde{K}_{i} B\left(0, T_{i-1}^{*}\right)}{2 \pi} \int_{\mathbb{R}}\left\{\left(\frac{F\left(0, T_{i}^{*}, T_{i-1}^{*}\right)}{\widetilde{K}_{i}}\right)^{R+\mathrm{i} u}\right. \\
& \times \exp \left(\int_{0}^{T_{i}^{*}} \int_{\mathbb{R}} e^{x \Lambda^{i-1}(s)}\left[\left(e^{(R+\mathrm{i} u) x \lambda\left(s, T_{i}^{*}\right)}-1\right)-(R+\mathrm{i} u)\left(e^{x \lambda\left(s, T_{i}^{*}\right)}-1\right)\right] F_{S}^{T^{*}}(d x) d s\right) \\
& \left.\times \exp \left(\int_{0}^{T_{i}^{*}} \frac{c_{s}}{2}(R+\mathrm{i} u)(R+\mathrm{i} u-1) \lambda^{2}\left(s, T_{i}^{*}\right) d s\right)\right\} \frac{d u}{(R+\mathrm{i} u)(R+\mathrm{i} u-1)} .
\end{aligned}
$$

Proof The time-0-price of the caplet with strike rate $K$ and maturity $T_{i}^{*}$ has the form

$$
\begin{aligned}
\operatorname{Cplt}_{0}\left(T_{i}^{*}, K\right) & =B\left(0, T_{i-1}^{*}\right) \mathbb{E}_{\mathbb{P}_{T_{i-1}^{*}}}\left[\left(e^{X_{T_{i}^{*}}}-\widetilde{K}_{i}\right)^{+}\right] \\
& =B\left(0, T_{i-1}^{*}\right) \mathbb{E}_{\mathbb{P}_{T_{i-1}^{*}}}\left[f\left(X_{T_{i}^{*}}\right)\right],
\end{aligned}
$$

where the function $f: \mathbb{R} \rightarrow \mathbb{R}^{+}$is defined by $f(x)=\left(e^{x}-\widetilde{K}_{i}\right)^{+}$.

Applying Theorem 2.2 in Eberlein et al. (2010) [8] (by the definition of $X_{T_{i}^{*}}$ we have $s=0$ here), we get

$$
\operatorname{Cplt}_{0}\left(T_{i}^{*}, K\right)=\frac{B\left(0, T_{i-1}^{*}\right)}{2 \pi} \int_{\mathbb{R}} M_{X_{T_{i}^{*}}}(R+\mathrm{i} u) \hat{f}(-u+\mathrm{i} R) d u,
$$

where the Fourier transform $\hat{f}$ is given by

$$
\hat{f}(-u+\mathrm{i} R)=\frac{\widetilde{K}_{i}^{1-R-\mathrm{i} u}}{(R+\mathrm{i} u)(R+\mathrm{i} u-1)}
$$


and the moment-generating function $M_{X_{T_{i}^{*}}}$ is given by

$$
\begin{aligned}
M_{X_{T_{i}^{*}}}(R+\mathrm{i} u)= & \mathbb{E}_{\mathbb{P}_{T_{i-1}^{*}}}\left[\exp \left((R+\mathrm{i} u) X_{T_{i}^{*}}\right)\right] \\
= & \left(F\left(0, T_{i}^{*}, T_{i-1}^{*}\right)\right)^{R+\mathrm{i} u} \exp \left((R+\mathrm{i} u) d\left(T_{i}^{*}, T_{i}^{*}\right)\right) \\
& \times \mathbb{E}_{\mathbb{P}_{T_{i-1}^{*}}}\left[\exp \left(\int_{0}^{T_{i}^{*}}(R+\mathrm{i} u) \lambda\left(s, T_{i}^{*}\right) d L_{s}^{T^{*}}\right)\right] .
\end{aligned}
$$

Making a change of measure, we find

$$
\begin{aligned}
& M_{X_{T_{i}^{*}}}(R+\mathrm{i} u)=\left(F\left(0, T_{i}^{*}, T_{i-1}^{*}\right)\right)^{R+\mathrm{i} u} \exp \left((R+\mathrm{i} u) d\left(T_{i}^{*}, T_{i}^{*}\right)\right) \\
& \times \frac{\mathbb{E}_{\mathbb{P}_{T^{*}}}\left[\exp \left(\int_{0}^{T_{i}^{*}}\left((R+\mathrm{i} u) \lambda\left(s, T_{i}^{*}\right)+\Lambda^{i-1}(s)\right) d L_{s}^{T^{*}}\right)\right]}{\mathbb{E}_{\mathbb{P}_{T^{*}}}\left[\exp \left(\int_{0}^{T_{i}^{*}} \Lambda^{i-1}(s) d L_{s}^{T^{*}}\right)\right]} .
\end{aligned}
$$

Using Proposition 8 in Eberlein and Kluge (2006) [6], we can prove easily that

$$
\begin{aligned}
M_{X_{T_{i}^{*}}}(R+\mathrm{i} u)= & \left(F\left(0, T_{i}^{*}, T_{i-1}^{*}\right)\right)^{R+\mathrm{i} u} \\
& \times \exp \left((R+\mathrm{i} u) \int_{0}^{T_{i}^{*}}\left[-\theta_{s}\left(\Lambda^{i}(s)\right)+\theta_{s}\left(\Lambda^{i-1}(s)\right)\right] d s\right) \\
& \times \frac{\exp \left(\int_{0}^{T_{i}^{*}} \theta_{s}\left((R+\mathrm{i} u) \lambda\left(s, T_{i}^{*}\right)+\Lambda^{i-1}(s)\right) d s\right)}{\exp \left(\int_{0}^{T_{i}^{*}} \theta_{s}\left(\Lambda^{i-1}(s)\right) d s\right)} \\
= & \left(F\left(0, T_{i}^{*}, T_{i-1}^{*}\right)\right)^{R+\mathrm{i} u} \exp \left(\int_{0}^{T_{i}^{*}} \theta_{s}\left((R+\mathrm{i} u) \lambda\left(s, T_{i}^{*}\right)+\Lambda^{i-1}(s)\right) d s\right) \\
& \times \exp \left(\int_{0}^{T_{i}^{*}}\left[(-R-\mathrm{i} u) \theta_{s}\left(\Lambda^{i}(s)\right)-(1-R-\mathrm{i} u) \theta_{s}\left(\Lambda^{i-1}(s)\right)\right] d s\right) .
\end{aligned}
$$

Taking into account the choice of the drift coefficient in (19), the cumulant function $\theta_{s}$ (see (9)) and the moment-generating function $M_{X_{T_{i}^{*}}}$, respectively, become

$$
\begin{aligned}
\theta_{s}(R+\mathrm{i} u)= & (R+\mathrm{i} u) \int_{\mathbb{R}}\left(\frac{e^{x(R+\mathrm{i} u)}-1}{R+\mathrm{i} u}-\frac{\left(e^{x \lambda\left(s, T_{1}^{*}\right)}-1\right)}{\lambda\left(s, T_{1}^{*}\right)}\right) F_{s}^{T^{*}}(d x) \\
& +\frac{c_{s}}{2}(R+\mathrm{i} u)\left(R+\mathrm{i} u-\lambda\left(s, T_{1}^{*}\right)\right)
\end{aligned}
$$

and

$$
M_{X_{i}^{*}}(R+\mathrm{i} u)=\left(F\left(0, T_{i}^{*}, T_{i-1}^{*}\right)\right)^{R+\mathrm{i} u} \exp \left(\int_{0}^{T_{i}^{*}} \frac{c_{s}}{2}(R+\mathrm{i} u)(R+\mathrm{i} u-1) \lambda^{2}\left(s, T_{i}^{*}\right) d s\right)
$$




$$
\begin{aligned}
& \times \exp \left(\int_{0}^{T_{i}^{*}} \int_{\mathbb{R}} e^{x \Lambda^{i-1}(s)}\left(e^{(R+\mathrm{i} u) x \lambda\left(s, T_{i}^{*}\right)}-1\right) F_{s}^{T^{*}}(d x) d s\right) \\
& \times \exp \left(-(R+\mathrm{i} u) \int_{0}^{T_{i}^{*}} \int_{\mathbb{R}} e^{x \Lambda^{i-1}(s)}\left(e^{x \lambda\left(s, T_{i}^{*}\right)}-1\right) F_{s}^{T^{*}}(d x) d s\right) .
\end{aligned}
$$

Finally, from (48) and (53) we conclude that

$$
\begin{aligned}
& \operatorname{Cplt}_{0}\left(T_{i}^{*}, K\right)=\frac{\widetilde{K}_{i} B\left(0, T_{i-1}^{*}\right)}{2 \pi} \int_{\mathbb{R}}\left\{\left(\frac{F\left(0, T_{i}^{*}, T_{i-1}^{*}\right)}{\widetilde{K}_{i}}\right)^{R+\mathrm{i} u}\right. \\
& \times \exp \left(\int_{0}^{T_{i}^{*}} \int_{\mathbb{R}} e^{x \Lambda^{i-1}(s)}\left[\left(e^{(R+\mathrm{i} u) x \lambda\left(s, T_{i}^{*}\right)}-1\right)-(R+\mathrm{i} u)\left(e^{x \lambda\left(s, T_{i}^{*}\right)}-1\right)\right] F_{s}^{T^{*}}(d x) d s\right) \\
& \left.\times \exp \left(\int_{0}^{T_{i}^{*}} \frac{c_{s}}{2}(R+\mathrm{i} u)(R+\mathrm{i} u-1) \lambda^{2}\left(s, T_{i}^{*}\right) d s\right)\right\} \frac{d u}{(R+\mathrm{i} u)(R+\mathrm{i} u-1)} .
\end{aligned}
$$

\section{Sensitivity Analysis}

\subsection{Greeks Computed by the Malliavin Approach}

In this section we present an application of the Malliavin calculus to the computation of Greeks within the Lévy forward process model. We refer to the literature, for example Di Nunno et al. (2008) [2] as well as Nualart (2006) [15] for details on the theoretical aspects of Malliavin calculus. Another important reference is Yablonski (2008) [19]. See also the Appendix for a short presentation of definitions and results used in the sequel. The forward process $F\left(t, T_{i}^{*}, T_{i-1}^{*}\right)$ under the forward measures $\mathbb{P}_{T_{i-1}^{*}}$ can be written as stochastic exponential

$$
F\left(t, T_{i}^{*}, T_{i-1}^{*}\right)=F\left(0, T_{i}^{*}, T_{i-1}^{*}\right) \mathscr{E}_{t}\left(Z\left(\cdot, T_{i}^{*}\right)\right)
$$

with

$$
Z\left(t, T_{i}^{*}\right)=\int_{0}^{t} \sqrt{c_{s}} \lambda\left(s, T_{i}^{*}\right) d W_{s}^{T_{i-1}^{*}}+\int_{0}^{t} \int_{\mathbb{R}}\left(e^{x \lambda\left(s, T_{i}^{*}\right)}-1\right) \widetilde{\mu}^{L^{T_{i-1}^{*}}}(d s, d x) .
$$

Expressed in a differential form we get the $\mathbb{P}_{T_{i-1}^{*}}$-dynamics

$$
\frac{d F\left(t, T_{i}^{*}, T_{i-1}^{*}\right)}{F\left(t-, T_{i}^{*}, T_{i-1}^{*}\right)}=\sqrt{c_{t}} \lambda\left(t, T_{i}^{*}\right) d W_{t}^{T_{i-1}^{*}}+\int_{\mathbb{R}}\left(e^{x \lambda\left(t, T_{i}^{*}\right)}-1\right) \widetilde{\mu}^{L^{T_{i-1}^{*}}}(d t, d x)
$$

where $F\left(t-, T_{i}^{*}, T_{i-1}^{*}\right)$ is the pathwise left limit of $F\left(\cdot, T_{i}^{*}, T_{i-1}^{*}\right)$ at the point $t$. 
As in the classical Malliavin calculus we are able to associate the solution of (57) with the process $Y\left(t, T_{i}^{*}, T_{i-1}^{*}\right):=\frac{\partial F\left(t, T_{i}^{*}, T_{i-1}^{*}\right)}{\partial F\left(0, T_{i}^{*}, T_{i-1}^{*}\right)}$; called the first variation process of $F\left(t, T_{i}^{*}, T_{i-1}^{*}\right)$. The following proposition provides a simpler expression for the Malliavin derivative operator $D_{r, 0}$ when applied to the forward process rates $F\left(t, T_{i}^{*}, T_{i-1}^{*}\right)$ (see Di Nunno et al. (2008) [2], Theorem 17.4 and Yablonski (2008) [19], Definition 17. for details). We will denote the domain of the operator $D_{r, 0}$ in $L^{2}(\Omega)$ by $\mathbb{D}^{1,2}$, meaning that $\mathbb{D}^{1,2}$ is the closure of the class of smooth random variables $\mathscr{S}$ (see (100) in the Appendix).

Proposition 4.1 Let $F\left(t, T_{i}^{*}, T_{i-1}^{*}\right)_{t \in\left[0, T^{*}\right]}$ be the solution of (57). Then $F\left(t, T_{i}^{*}\right.$, $\left.T_{i-1}^{*}\right) \in \mathbb{D}^{1,2}$ and the Malliavin derivative is given by

$$
\begin{aligned}
& D_{r, 0} F\left(t, T_{i}^{*}, T_{i-1}^{*}\right) \\
& \quad=Y\left(t, T_{i}^{*}, T_{i-1}^{*}\right) Y\left(r-, T_{i}^{*}, T_{i-1}^{*}\right)^{-1} F\left(r-, T_{i}^{*}, T_{i-1}^{*}\right) \lambda\left(r, T_{i}^{*}\right) \sqrt{c_{r}} \mathbf{1}_{\{r \leq t\}} .
\end{aligned}
$$

\subsubsection{Variation in the Initial Forward Price}

In this section, we provide an expression for the Delta, the partial derivative of the expectation $\operatorname{Cplt}_{0}\left(T_{i}^{*}, K\right)$ with respect to the initial condition $F\left(0, T_{i}^{*}, T_{i-1}^{*}\right)$ which is given by

$$
\Delta\left(F\left(0, T_{i}^{*}, T_{i-1}^{*}\right)\right)=\frac{\partial C p l t_{0}\left(T_{i}^{*}, K\right)}{\partial F\left(0, T_{i}^{*}, T_{i-1}^{*}\right)} .
$$

The derivative with respect to the initial LIBOR rate is then an easy consequence.

$$
\begin{aligned}
\Delta\left(L\left(0, T_{i}^{*}\right)\right) & =\frac{\partial \operatorname{Cplt}_{0}\left(T_{i}^{*}, K\right)}{\partial L\left(0, T_{i}^{*}\right)} \\
& =\Delta\left(F\left(0, T_{i}^{*}, T_{i-1}^{*}\right)\right) \frac{\partial F\left(0, T_{i}^{*}, T_{i-1}^{*}\right)}{\partial L\left(0, T_{i}^{*}\right)} \\
& =\delta_{i}^{*} \Delta\left(F\left(0, T_{i}^{*}, T_{i-1}^{*}\right)\right),
\end{aligned}
$$

since

$$
L\left(0, T_{i}^{*}\right)=\frac{1}{\delta_{i}^{*}}\left(F\left(0, T_{i}^{*}, T_{i-1}^{*}\right)-1\right) .
$$

Let us define the set

$$
\widetilde{T}_{i}=\left\{h_{i} \in L^{2}\left(\left[0, T_{i}^{*}\right]\right): \int_{0}^{T_{i}^{*}} h_{i}(u) d u=1\right\} .
$$


Proposition 4.2 For all functions $h_{i} \in \widetilde{T}_{i}$, we have

$$
\begin{aligned}
\Delta\left(F\left(0, T_{i}^{*}, T_{i-1}^{*}\right)\right)= & \frac{B\left(0, T_{i-1}^{*}\right)}{F\left(0, T_{i}^{*}, T_{i-1}^{*}\right)} \mathbb{E}_{\mathbb{P}_{T^{*}}}\left[\left(F\left(T_{i}^{*}, T_{i}^{*}, T_{i-1}^{*}\right)-\widetilde{K}_{i}\right)^{+}\right. \\
& \times \exp \left(\int_{0}^{T_{i}^{*}} \Lambda^{i-1}(s) d L_{s}^{T^{*}}-\int_{0}^{T_{i}^{*}} \theta_{s}\left(\Lambda^{i-1}(s)\right) d s\right) \\
& \left.\times\left(\int_{0}^{T_{i}^{*}} \frac{h_{i}(u) d W_{u}^{T^{*}}}{\lambda\left(u, T_{i}^{*}\right) \sqrt{c_{u}}}-\int_{0}^{T_{i}^{*}} \frac{h_{i}(u) \Lambda^{i-1}(u) d u}{\lambda\left(u, T_{i}^{*}\right)}\right)\right] .
\end{aligned}
$$

Proof We consider a more general payoff of the form $H\left(F\left(T_{i}^{*}, T_{i}^{*}, T_{i-1}^{*}\right)\right)$ such that $H: \mathbb{R} \longrightarrow \mathbb{R}$ is a locally integrable function satisfying

$$
\mathbb{E}_{\mathbb{P}_{T_{i-1}^{*}}}\left[H\left(F\left(T_{i}^{*}, T_{i}^{*}, T_{i-1}^{*}\right)\right)^{2}\right]<\infty
$$

First, assume that $H$ is a continuously differentiable function with compact support. Then we can differentiate inside the expectation and get

$$
\begin{aligned}
\Delta_{H}\left(F\left(0, T_{i}^{*}, T_{i-1}^{*}\right)\right) & :=\frac{\partial \mathbb{E}_{\mathbb{P}_{T_{i-1}^{*}}}\left[H\left(F\left(T_{i}^{*}, T_{i}^{*}, T_{i-1}^{*}\right)\right)\right]}{\partial F\left(0, T_{i}^{*}, T_{i-1}^{*}\right)} \\
& =\mathbb{E}_{\mathbb{P}_{T_{i-1}^{*}}}\left[H^{\prime}\left(F\left(T_{i}^{*}, T_{i}^{*}, T_{i-1}^{*}\right)\right) \frac{\left.\partial F\left(T_{i}^{*}, T_{i}^{*}, T_{i-1}^{*}\right)\right]}{\partial F\left(0, T_{i}^{*}, T_{i-1}^{*}\right)}\right] \\
& =\mathbb{E}_{\mathbb{P}_{T_{i-1}^{*}}}\left[H^{\prime}\left(F\left(T_{i}^{*}, T_{i}^{*}, T_{i-1}^{*}\right)\right) Y\left(T_{i}^{*}, T_{i}^{*}, T_{i-1}^{*}\right)\right] .
\end{aligned}
$$

Using Proposition 4.1 we find for any $h_{i} \in \widetilde{T}_{i}$

$$
Y\left(T_{i}^{*}, T_{i}^{*}, T_{i-1}^{*}\right)=\int_{0}^{T_{i}^{*}} D_{u, 0} F\left(T_{i}^{*}, T_{i}^{*}, T_{i-1}^{*}\right) \frac{h_{i}(u) Y\left(u-, T_{i}^{*}, T_{i-1}^{*}\right) d u}{F\left(u-, T_{i}^{*}, T_{i-1}^{*}\right) \lambda\left(u, T_{i}^{*}\right) \sqrt{c_{u}}} .
$$

From the chain rule (see Yablonski (2008) [19], Proposition 4.8) we find

$$
\begin{aligned}
\Delta_{H}\left(F\left(0, T_{i}^{*}, T_{i-1}^{*}\right)\right)=\mathbb{E}_{\mathbb{P}_{i-1}^{*}}\left[\int_{0}^{T_{i}^{*}} H^{\prime}\left(F\left(T_{i}^{*}, T_{i}^{*}, T_{i-1}^{*}\right)\right) D_{u, 0} F\left(T_{i}^{*}, T_{i}^{*}, T_{i-1}^{*}\right)\right. \\
\left.\times \frac{h_{i}(u) Y\left(u-, T_{i}^{*}, T_{i-1}^{*}\right) d u}{F\left(u-, T_{i}^{*}, T_{i-1}^{*}\right) \lambda\left(u, T_{i}^{*}\right) \sqrt{c_{u}}}\right] \\
=\mathbb{E}_{\mathbb{P}_{T_{i-1}^{*}}\left[\int_{0}^{T_{i}^{*}} D_{u, 0} H\left(F\left(T_{i}^{*}, T_{i}^{*}, T_{i-1}^{*}\right)\right)\right.} \\
\left.\times \frac{h_{i}(u) Y\left(u-, T_{i}^{*}, T_{i-1}^{*}\right) d u}{F\left(u-, T_{i}^{*}, T_{i-1}^{*}\right) \lambda\left(u, T_{i}^{*}\right) \sqrt{c_{u}}}\right]
\end{aligned}
$$




$$
\begin{aligned}
=\mathbb{E}_{\mathbb{P}_{T_{i-1}^{*}}}\left[\int_{0}^{T_{i}^{*}} \int_{\mathbb{R}} D_{u, x} H\left(F\left(T_{i}^{*}, T_{i}^{*}, T_{i-1}^{*}\right)\right)\right. \\
\left.\times \frac{h_{i}(u) Y\left(u-, T_{i}^{*}, T_{i-1}^{*}\right) d u \delta_{0}(d x)}{F\left(u-, T_{i}^{*}, T_{i-1}^{*}\right) \lambda\left(u, T_{i}^{*}\right) \sqrt{c_{u}}}\right],
\end{aligned}
$$

where $\delta_{0}(d x)$ is the Dirac measure at 0 .

By the definition of the Skorohod integral $\delta(\cdot)$ (see Yablonski (2008) [19], Sect. 5), we reach

$$
\begin{aligned}
& \Delta_{H}\left(F\left(0, T_{i}^{*}, T_{i-1}^{*}\right)\right) \\
& \quad=\mathbb{E}_{\mathbb{P}_{T_{i-1}^{*}}}\left[H\left(F\left(T_{i}^{*}, T_{i}^{*}, T_{i-1}^{*}\right)\right) \delta\left(\frac{h_{i}(\cdot) Y\left(\cdot-, T_{i}^{*}, T_{i-1}^{*}\right) \delta_{0}(\cdot)}{F\left(\cdot-, T_{i}^{*}, T_{i-1}^{*}\right) \lambda\left(\cdot, T_{i}^{*}\right) \sqrt{c}}\right)\right] .
\end{aligned}
$$

However, the stochastic process

$$
\left(\frac{h_{i}(u) Y\left(u-, T_{i}^{*}, T_{i-1}^{*}\right)}{F\left(u-, T_{i}^{*}, T_{i-1}^{*}\right) \lambda\left(u, T_{i}^{*}\right) \sqrt{c_{u}}}\right)_{0 \leq u \leq T_{i}^{*}}
$$

is a predictable process, thus the Skorohod integral coincides with the Itô stochastic integral and we get

$$
\begin{aligned}
& \Delta_{H}\left(F\left(0, T_{i}^{*}, T_{i-1}^{*}\right)\right) \\
& \quad=\mathbb{E}_{\mathbb{P}_{T_{i-1}^{*}}}\left[H\left(F\left(T_{i}^{*}, T_{i}^{*}, T_{i-1}^{*}\right)\right) \int_{0}^{T_{i}^{*}} \frac{h_{i}(u) Y\left(u-, T_{i}^{*}, T_{i-1}^{*}\right) d W_{u}^{T_{i-1}^{*}}}{F\left(u-, T_{i}^{*}, T_{i-1}^{*}\right) \lambda\left(u, T_{i}^{*}\right) \sqrt{c_{u}}}\right] .
\end{aligned}
$$

By Lemma 12.28. p. 208 in Di Nunno et al. (2008) [2] the result (70) holds for any locally integrable function $H$ such that

$$
\mathbb{E}_{\mathbb{P}_{T_{i-1}^{*}}}\left[H\left(F\left(T_{i}^{*}, T_{i}^{*}, T_{i-1}^{*}\right)\right)^{2}\right]<\infty
$$

In particular, if one takes

$$
H\left(F\left(T_{i}^{*}, T_{i}^{*}, T_{i-1}^{*}\right)\right)=B\left(0, T_{i-1}^{*}\right)\left(F\left(T_{i}^{*}, T_{i}^{*}, T_{i-1}^{*}\right)-\widetilde{K}_{i}\right)^{+},
$$

we can express the derivatives of the expectation $\operatorname{Cplt}_{0}\left(T_{i}^{*}, K, \delta_{i}^{*}\right)$ with respect to the initial condition $F\left(0, T_{i}^{*}, T_{i-1}^{*}\right)$ in the form of a weighted expectation as follows

$$
\begin{aligned}
\Delta\left(F\left(0, T_{i}^{*}, T_{i-1}^{*}\right)\right)=B\left(0, T_{i-1}^{*}\right) \mathbb{E}_{\mathbb{P}_{T_{i-1}^{*}}}[ & \left(F\left(T_{i}^{*}, T_{i}^{*}, T_{i-1}^{*}\right)-\widetilde{K}_{i}\right)^{+} \\
& \left.\times \int_{0}^{T_{i}^{*}} \frac{h_{i}(u) Y\left(u-, T_{i}^{*}, T_{i-1}^{*}\right) d W_{u}^{T_{i-1}^{*}}}{\lambda\left(u, T_{i}^{*}\right) \sqrt{c_{u}} F\left(u-, T_{i}^{*}, T_{i-1}^{*}\right)}\right] .
\end{aligned}
$$


We show easily that

$$
Y\left(u-, T_{i}^{*}, T_{i-1}^{*}\right)=\frac{F\left(u-, T_{i}^{*}, T_{i-1}^{*}\right)}{F\left(0, T_{i}^{*}, T_{i-1}^{*}\right)}
$$

hence

$$
\begin{aligned}
& \Delta\left(F\left(0, T_{i}^{*}, T_{i-1}^{*}\right)\right) \\
& \quad=\frac{B\left(0, T_{i-1}^{*}\right)}{F\left(0, T_{i}^{*}, T_{i-1}^{*}\right)} \mathbb{E}_{\mathbb{P}_{T_{i-1}^{*}}}\left[\left(F\left(T_{i}^{*}, T_{i}^{*}, T_{i-1}^{*}\right)-\widetilde{K}_{i}\right)^{+} \int_{0}^{T_{i}^{*}} \frac{h_{i}(u) d W_{u}^{T_{i-1}^{*}}}{\lambda\left(u, T_{i}^{*}\right) \sqrt{c_{u}}}\right] .
\end{aligned}
$$

In accordance with (25) we can write

$$
W_{t}^{T_{i-1}^{*}}=W_{t}^{T^{*}}-\int_{0}^{t} \Lambda^{i-1}(s) \sqrt{c_{s}} d s .
$$

By making a measure change using the fact (see (40)) that

$$
\left.\frac{d \mathbb{P}_{T_{i-1}^{*}}}{d \mathbb{P}_{T^{*}}}\right|_{\mathscr{F}_{T_{i}^{*}}}=\exp \left(\int_{0}^{T_{i}^{*}} \Lambda^{i-1}(s) d L_{s}^{T^{*}}-\int_{0}^{T_{i}^{*}} \theta_{s}\left(\Lambda^{i-1}(s)\right) d s\right),
$$

we end up with

$$
\begin{aligned}
\Delta\left(F\left(0, T_{i}^{*}, T_{i-1}^{*}\right)\right)= & \frac{B\left(0, T_{i-1}^{*}\right)}{F\left(0, T_{i}^{*}, T_{i-1}^{*}\right)} \mathbb{E}_{\mathbb{P}_{T^{*}}}\left[\left(F\left(T_{i}^{*}, T_{i}^{*}, T_{i-1}^{*}\right)-\widetilde{K}_{i}\right)^{+}\right. \\
& \times \exp \left(\int_{0}^{T_{i}^{*}} \Lambda^{i-1}(s) d L_{s}^{T^{*}}-\int_{0}^{T_{i}^{*}} \theta_{s}\left(\Lambda^{i-1}(s)\right) d s\right) \\
& \left.\times\left(\int_{0}^{T_{i}^{*}} \frac{h_{i}(u) d W_{u}^{T^{*}}}{\lambda\left(u, T_{i}^{*}\right) \sqrt{c_{u}}}-\int_{0}^{T_{i}^{*}} \frac{h_{i}(u) \Lambda^{i-1}(u)}{\lambda\left(u, T_{i}^{*}\right)} d u\right)\right] .
\end{aligned}
$$

\subsection{Greeks Computed by the Fourier-Based Valuation Method}

Thanks to the Fourier-based valuation formula obtained in (45) and the structure of the forward process model as an exponential semimartingale, we can calculate readily the Greeks. We focus on the variation to the initial condition, i.e. Delta.

Proposition 4.3 Suppose there is a real number $R \in(1,1+\varepsilon)$ such that the moment-generating function of $X_{T_{i}^{*}}$ with respect to $\mathbb{P}_{T_{i-1}^{*}}$ is finite at $R$, i.e. $M_{X_{T_{i}^{*}}}(R)<$ $\infty$, then 


$$
\begin{aligned}
\Delta\left(F\left(0, T_{i}^{*}, T_{i-1}^{*}\right)\right)= & \frac{B\left(0, T_{i-1}^{*}\right)}{2 \pi} \int_{\mathbb{R}}\left\{\left(\frac{F\left(0, T_{i}^{*}, T_{i-1}^{*}\right)}{\widetilde{K}_{i}}\right)^{R+\mathrm{i} u-1}\right. \\
& \times \exp \left(\int_{0}^{T_{i}^{*}} \int_{\mathbb{R}} e^{x \Lambda^{i-1}(s)}\left(e^{(R+\mathrm{i} u) x \lambda\left(s, T_{i}^{*}\right)}-1\right) F_{s}^{T^{*}}(d x) d s\right) \\
& \times \exp \left(-\int_{0}^{T_{i}^{*}} \int_{\mathbb{R}} e^{x \Lambda^{i-1}(s)}(R+\mathrm{i} u)\left(e^{x \lambda\left(s, T_{i}^{*}\right)}-1\right) F_{s}^{T^{*}}(d x) d s\right) \\
& \left.\times \exp \left(\int_{0}^{T_{i}^{*}} \frac{c_{s}}{2}(R+\mathrm{i} u)(R+\mathrm{i} u-1) \lambda^{2}\left(s, T_{i}^{*}\right) d s\right)\right\} \frac{d u}{R+\mathrm{i} u-1} .
\end{aligned}
$$

Proof Based on the Sect. 4 in Eberlein et al. (2010) [8], this proposition can be shown easily.

\subsection{Examples}

\subsubsection{Variance Gamma Process (VG)}

We suppose that the jump component of the driving process $L^{T^{*}}$ (see (8)) is described by a Variance Gamma process with the Lévy density $v$ given by

$$
v(d x)=F_{V G}(x) d x
$$

such that

$$
F_{V G}(x):=\frac{1}{\eta|x|} \exp \left(\frac{\theta}{\sigma^{2}} x-\frac{1}{\sigma} \sqrt{\frac{2}{\eta}+\frac{\theta^{2}}{\sigma^{2}}}|x|\right)
$$

where $(\theta, \sigma, \eta)$ are the parameters such that $\theta \in \mathbb{R}, \sigma>0$ and $\eta>0$.

Let us put $B=\frac{\theta}{\sigma^{2}}$ and $C=\frac{1}{\sigma} \sqrt{\frac{2}{\eta}+\frac{\theta^{2}}{\sigma^{2}}}$ and get

$$
F_{V G}(x)=\frac{\exp (B x-C|x|)}{\eta|x|} .
$$

In this case, the moment-generating function $M_{X_{T_{i}^{*}}}$ is given by

$$
M_{X_{T_{i}^{*}}}(z)=\left(F\left(0, T_{i}^{*}, T_{i-1}^{*}\right)\right)^{z} \exp \left(\int_{0}^{T_{i}^{*}}\left(\frac{c_{s} z}{2}(z-1) \lambda^{2}\left(s, T_{i}^{*}\right)+I^{V G}(s, z)\right) d s\right),
$$

where the generalized integral $I^{V G}(s, z)$ is given by 


$$
\begin{aligned}
I^{V G}(s, z):= & \int_{\mathbb{R}}\left(e^{x\left(z \lambda\left(s, T_{i}^{*}\right)+\Lambda^{i-1}(s)\right)}-e^{x \Lambda^{i-1}(s)}\right) F_{V G}(x) d x \\
& -\int_{\mathbb{R}} z\left(e^{x \Lambda^{i}(s)}-e^{x \Lambda^{i-1}(s)}\right) F_{V G}(x) d x .
\end{aligned}
$$

Now substituting $F_{V G}(x)$ by its explicit expression we get

$$
\begin{aligned}
I^{V G}(s, z)= & \int_{\mathbb{R}}\left(e^{x\left(z \lambda\left(s, T_{i}^{*}\right)+\Lambda^{i-1}(s)\right)}-e^{x \Lambda^{i-1}(s)}\right) \exp (B x-C|x|) \frac{d x}{\eta|x|} \\
& -\int_{\mathbb{R}} z\left(e^{x \Lambda^{i}(s)}-e^{x \Lambda^{i-1}(s)}\right) \exp (B x-C|x|) \frac{d x}{\eta|x|} \\
= & \int_{0}^{+\infty}\left(e^{x\left(z \lambda\left(s, T_{i}^{*}\right)+\Lambda^{i-1}(s)\right)}-e^{x \Lambda^{i-1}(s)}\right) \exp (B x-C x) \frac{d x}{\eta x} \\
& -\int_{0}^{+\infty} z\left(e^{x \Lambda^{i}(s)}-e^{x \Lambda^{i-1}(s)}\right) \exp (B x-C x) \frac{d x}{\eta x} \\
& -\int_{-\infty}^{0}\left(e^{x\left(z \lambda\left(s, T_{i}^{*}\right)+\Lambda^{i-1}(s)\right)}-e^{x \Lambda^{i-1}(s)}\right) \exp (B x+C x) \frac{d x}{\eta x} \\
& +\int_{-\infty}^{0} z\left(e^{x \Lambda^{i}(s)}-e^{x \Lambda^{i-1}(s)}\right) \exp (B x+C x) \frac{d x}{\eta x},
\end{aligned}
$$

or

$$
\begin{aligned}
& I^{V G}(s, z)=\int_{0}^{+\infty}\left[\frac{e^{\left(z \lambda\left(s, T_{i}^{*}\right)+\Lambda^{i-1}(s)+B-C\right) x}-e^{\left(\Lambda^{i-1}(s)+B-C\right) x}}{\eta x}\right] d x \\
& -\int_{0}^{+\infty}\left[z \frac{e^{\left(\Lambda^{i}(s)+B-C\right) x}-e^{\left(\Lambda^{i-1}(s)+B-C\right) x}}{\eta x}\right] d x \\
& -\int_{-\infty}^{0}\left[\frac{e^{\left(z \lambda\left(s, T_{i}^{*}\right)+\Lambda^{i-1}(s)+B+C\right) x}-e^{\left(\Lambda^{i-1}(s)+B+C\right) x}}{\eta x}\right] d x \\
& +\int_{-\infty}^{0}\left[z \frac{e^{\left(\Lambda^{i}(s)+B+C\right) x}-e^{\left(\Lambda^{i-1}(s)+B+C\right) x}}{\eta x}\right] d x \\
& =\int_{0}^{+\infty}\left[\frac{e^{\left(z \lambda\left(s, T_{i}^{*}\right)+\Lambda^{i-1}(s)+B-C\right) x}-e^{\left(\Lambda^{i-1}(s)+B-C\right) x}}{\eta x}\right] d x \\
& -\int_{0}^{+\infty}\left[z \frac{e^{\left(\Lambda^{i}(s)+B-C\right) x}-e^{\left(\Lambda^{i-1}(s)+B-C\right) x}}{\eta x}\right] d x \\
& +\int_{0}^{+\infty}\left[\frac{e^{-\left(z \lambda\left(s, T_{i}^{*}\right)+\Lambda^{i-1}(s)+B+C\right) x}-e^{-\left(\Lambda^{i-1}(s)+B+C\right) x}}{\eta x}\right] d x \\
& -\int_{0}^{+\infty}\left[z \frac{e^{-\left(\Lambda^{i}(s)+B+C\right) x}-e^{-\left(\Lambda^{i-1}(s)+B+C\right) x}}{\eta x}\right] d x .
\end{aligned}
$$


Using the notations

$$
\begin{aligned}
\alpha_{i}(s, z) & =-\left(z \lambda\left(s, T_{i}^{*}\right)+\Lambda^{i-1}(s)+B-C\right), \\
\beta_{i}(s) & =-\left(\Lambda^{i-1}(s)+B-C\right), \\
\gamma_{i}(s) & =-\left(\Lambda^{i}(s)+B-C\right),
\end{aligned}
$$

we end up with

$$
\begin{aligned}
I^{V G}(s, z)= & \int_{0}^{+\infty}\left[\frac{e^{-\alpha_{i}(s, z) x}-e^{-\beta_{i}(s) x}}{x}-z \frac{e^{-\gamma_{i}(s) x}-e^{-\beta_{i}(s) x}}{x}\right] d x \\
& +\int_{0}^{+\infty}\left[\frac{e^{-\left(2 C-\alpha_{i}(s, z)\right) x}-e^{-\left(2 C-\beta_{i}(s)\right) x}}{x}-z \frac{e^{-\left(2 C-\gamma_{i}(s)\right) x}-e^{-\left(2 C-\beta_{i}(s)\right) x}}{x}\right] d x .
\end{aligned}
$$

Using Frullani's integral (see for details Ostrowski (1949) [16]), we can show that, if $\alpha \in \mathbb{C}$ and $\beta \in \mathbb{C}$ such that $\mathscr{R} e(\alpha)>0, \mathscr{R} e(\beta)>0$ and $\frac{\beta}{\alpha} \in \mathbb{C} \backslash \mathbb{R}^{-}$where $\mathbb{R}^{-}=$ ]$-\infty ; 0]$,

$$
I_{(\alpha, \beta)}:=\int_{0}^{+\infty} \frac{e^{-\alpha x}-e^{-\beta x}}{x} d x=\log \left(\frac{\beta}{\alpha}\right),
$$

where $\log$ is the principal value of the logarithm. Consequently

$$
\begin{aligned}
I^{V G}(s, z)= & \log \left(\frac{\beta_{i}(s)}{\alpha_{i}(s, z)}\right)-z \log \left(\frac{\beta_{i}(s)}{\gamma_{i}(s)}\right) \\
& +\log \left(\frac{2 C-\beta_{i}(s)}{2 C-\alpha_{i}(s, z)}\right)-z \log \left(\frac{2 C-\beta_{i}(s)}{2 C-\gamma_{i}(s)}\right) \\
= & \log \left(\frac{\beta_{i}(s)}{\alpha_{i}(s, z)}\right)+\log \left(\frac{2 C-\beta_{i}(s)}{2 C-\alpha_{i}(s, z)}\right) \\
& -z\left(\log \left(\frac{\beta_{i}(s)}{\gamma_{i}(s)}\right)+\log \left(\frac{2 C-\beta_{i}(s)}{2 C-\gamma_{i}(s)}\right)\right) \\
= & \log \left(\frac{\beta_{i}(s)\left(2 C-\beta_{i}(s)\right)}{\alpha_{i}(s, z)\left(2 C-\alpha_{i}(s, z)\right)}\right)-z \log \left(\frac{\beta_{i}(s)\left(2 C-\beta_{i}(s)\right)}{\gamma_{i}(s)\left(2 C-\gamma_{i}(s)\right)}\right) .
\end{aligned}
$$

The moment-generating function $M_{X_{T_{i}^{*}}}$ becomes

$$
\begin{aligned}
M_{X_{T_{i}^{*}}}(z)= & \left(F\left(0, T_{i}^{*}, T_{i-1}^{*}\right)\right)^{z} \exp \left(\int_{0}^{T_{i}^{*}} \frac{c_{s} z}{2}(z-1) \lambda^{2}\left(s, T_{i}^{*}\right) d s\right) \\
& \times \exp \left(\int_{0}^{T_{i}^{*}} \log \left(\frac{\beta_{i}(s)\left(2 C-\beta_{i}(s)\right)}{\alpha_{i}(s, z)\left(2 C-\alpha_{i}(s, z)\right)}\right) d s\right) \\
& \times \exp \left(-\int_{0}^{T_{i}^{*}} z \log \left(\frac{\beta_{i}(s)\left(2 C-\beta_{i}(s)\right)}{\gamma_{i}(s)\left(2 C-\gamma_{i}(s)\right)}\right) d s\right)
\end{aligned}
$$


or

$$
\begin{aligned}
M_{X_{T_{i}^{*}}}(R+\mathrm{i} u)= & \left(F\left(0, T_{i}^{*}, T_{i-1}^{*}\right)\right)^{R+\mathrm{i} u} \\
& \times \exp \left(\int_{0}^{T_{i}^{*}} \frac{c_{s}}{2}(R+\mathrm{i} u)(R+\mathrm{i} u-1) \lambda^{2}\left(s, T_{i}^{*}\right) d s\right) \\
& \times \exp \left(\int_{0}^{T_{i}^{*}} \log \left(\frac{\beta_{i}(s)\left(2 C-\beta_{i}(s)\right)}{\alpha_{i}(s, R+\mathrm{i} u)\left(2 C-\alpha_{i}(s, R+\mathrm{i} u)\right)}\right) d s\right) \\
& \times \exp \left(-\int_{0}^{T_{i}^{*}}(R+\mathrm{i} u) \log \left(\frac{\beta_{i}(s)\left(2 C-\beta_{i}(s)\right)}{\gamma_{i}(s)\left(2 C-\gamma_{i}(s)\right)}\right) d s\right) .
\end{aligned}
$$

The valuation formula becomes

$$
\begin{aligned}
\operatorname{Cplt}_{0}\left(T_{i}^{*}, K\right)= & \frac{B\left(0, T_{i-1}^{*}\right)}{2 \pi} \int_{\mathbb{R}} \frac{\widetilde{K}_{i}^{1-R-\mathrm{i} u} M_{X_{T_{i}^{*}}}(R+\mathrm{i} u)}{(R+\mathrm{i} u)(R+\mathrm{i} u-1)} d u \\
= & \frac{\widetilde{K}_{i} B\left(0, T_{i-1}^{*}\right)}{2 \pi} \int_{\mathbb{R}}\left\{\left(\frac{F\left(0, T_{i}^{*}, T_{i-1}^{*}\right)}{\widetilde{K}_{i}}\right){ }^{R+\mathrm{i} u}\right. \\
& \times \exp \left(\int_{0}^{T_{i}^{*}} \frac{c_{s}}{2}(R+\mathrm{i} u)(R+\mathrm{i} u-1) \lambda^{2}\left(s, T_{i}^{*}\right) d s\right) \\
& \times \exp \left(\int_{0}^{T_{i}^{*}} \log \left(\frac{\beta_{i}(s)\left(2 C-\beta_{i}(s)\right)}{\alpha_{i}(s, R+\mathrm{i} u)\left(2 C-\alpha_{i}(s, R+\mathrm{i} u)\right)}\right) d s\right) \\
& \left.\times \exp \left(-\int_{0}^{T_{i}^{*}}(R+\mathrm{i} u) \log \left(\frac{\beta_{i}(s)\left(2 C-\beta_{i}(s)\right)}{\gamma_{i}(s)\left(2 C-\gamma_{i}(s)\right)}\right) d s\right)\right\} \frac{d u}{(R+\mathrm{i} u)(R+\mathrm{i} u-1)} .
\end{aligned}
$$

The Delta is given by

$$
\begin{aligned}
\Delta\left(F\left(0, T_{i}^{*}, T_{i-1}^{*}\right)\right)= & \frac{B\left(0, T_{i-1}^{*}\right)}{2 \pi} \int_{\mathbb{R}}\left\{\left(\frac{F\left(0, T_{i}^{*}, T_{i-1}^{*}\right)}{\widetilde{K}_{i}}\right)^{R+\mathrm{i} u-1}\right. \\
& \times \exp \left(\int_{0}^{T_{i}^{*}} \frac{c_{s}}{2}(R+\mathrm{i} u)(R+\mathrm{i} u-1) \lambda^{2}\left(s, T_{i}^{*}\right) d s\right) \\
& \times \exp \left(\int_{0}^{T_{i}^{*}} \log \left(\frac{\beta_{i}(s)\left(2 C-\beta_{i}(s)\right)}{\alpha_{i}(s, R+\mathrm{i} u)\left(2 C-\alpha_{i}(s, R+\mathrm{i} u)\right)}\right) d s\right) \\
& \left.\times \exp \left(-\int_{0}^{T_{i}^{*}}(R+\mathrm{i} u) \log \left(\frac{\beta_{i}(s)\left(2 C-\beta_{i}(s)\right)}{\gamma_{i}(s)\left(2 C-\gamma_{i}(s)\right)}\right) d s\right)\right\} \frac{d u}{R+\mathrm{i} u-1} .
\end{aligned}
$$

\subsubsection{Inhomogeneous Gamma Process (IGP)}

We suppose that the jump component of the driving process $L^{T^{*}}$, is described by an inhomogeneous Gamma process (IGP), which has been introduced by Berman (1981) [1] as follows 
Definition 4.4 Let $A(t)$ be a nondecreasing function from $\mathbb{R}^{+} \longrightarrow \mathbb{R}^{+}$and $B>0$. A Gamma process with shape function $A$ and scale parameter $B$ is a stochastic process $\left(L_{t}\right)_{t \geq 0}$ on $\mathbb{R}^{+}$such that:

1. $L_{0}=0$;

2. Independent increments: for every increasing sequence of time points $t_{0}, \ldots, t_{n}$, the random variables $L_{t_{0}}, L_{t_{1}}-L_{t_{0}}, \ldots, L_{t_{n}}-L_{t_{n-1}}$ are independent;

3. for $0 \leq s<t$, the distribution of the random variable $L_{t}-L_{s}$ is given by the Gamma distribution $\Gamma(A(t)-A(s) ; B)$.

We suppose that the shape function $A$ is differentiable, hence we can write

$$
A(t)=A(0)+\int_{0}^{t} \dot{A}(s) d s
$$

for all $t \in \mathbb{R}^{+}$where $\dot{A}$ denotes the derivative of $A$. In this case, the Lévy density of the Gamma process $L$ is given by

$$
F_{s}^{G}(x)=\dot{A}(s) \frac{e^{-B x}}{x} \mathbf{1}_{\{x>0\}} .
$$

The moment-generating function (53) has the form

$$
\begin{aligned}
M_{X_{T_{i}^{*}}}(z)= & \left(F\left(0, T_{i}^{*}, T_{i-1}^{*}\right)\right)^{z} \exp \left(\int_{0}^{T_{i}^{*}} \frac{c_{s} z}{2}(z-1) \lambda^{2}\left(s, T_{i}^{*}\right) d s\right) \\
& \times \exp \left(\int_{0}^{T_{i}^{*}} \int_{\mathbb{R}} e^{x \Lambda^{i-1}(s)}\left[\left(e^{z x \lambda\left(s, T_{i}^{*}\right)}-1\right)-z\left(e^{x \lambda\left(s, T_{i}^{*}\right)}-1\right)\right] F_{s}^{G}(x) d x d s\right) \\
= & \left(F\left(0, T_{i}^{*}, T_{i-1}^{*}\right)\right)^{z} \exp \left(\int_{0}^{T_{i}^{*}} \frac{c_{s} z}{2}(z-1) \lambda^{2}\left(s, T_{i}^{*}\right) d s\right) \\
& \times \exp \left(\int_{0}^{T_{i}^{*}} \dot{A}(s) \int_{\mathbb{R}} e^{x \Lambda^{i-1}(s)}\left(e^{z x \lambda\left(s, T_{i}^{*}\right)}-1\right) \frac{e^{-B x}}{x} \mathbf{1}_{\{x>0\}} d x d s\right) \\
& \times \exp \left(-z \int_{0}^{T_{i}^{*}} \dot{A}(s) \int_{\mathbb{R}} e^{x \Lambda^{i-1}(s)}\left(e^{x \lambda\left(s, T_{i}^{*}\right)}-1\right) \frac{e^{-B x}}{x} \mathbf{1}_{\{x>0\}} d x d s\right) \\
= & \left(F\left(0, T_{i}^{*}, T_{i-1}^{*}\right)\right)^{z} \exp \left(\int_{0}^{T_{i}^{*}}\left(\frac{c_{s} z}{2}(z-1) \lambda^{2}\left(s, T_{i}^{*}\right)+\dot{A}(s) I^{G}(s, z)\right) d s\right),
\end{aligned}
$$

where

$$
\begin{aligned}
I^{G}(s, z)= & \int_{0}^{+\infty} \frac{e^{\left(z \lambda\left(s, T_{i}^{*}\right)+\Lambda^{i-1}(s)-B\right) x}-e^{\left(\Lambda^{i-1}(s)-B\right) x}}{x} d x \\
& -\int_{0}^{+\infty} z \frac{e^{\left(\Lambda^{i}(s)-B\right) x}-e^{\left(\Lambda^{i-1}(s)-B\right) x}}{x} d x .
\end{aligned}
$$


Setting

$$
\begin{aligned}
\alpha_{i}(s, z) & =-\left(z \lambda\left(s, T_{i}^{*}\right)+\Lambda^{i-1}(s)-B\right), \\
\beta_{i}(s) & =-\left(\Lambda^{i-1}(s)-B\right) \\
\gamma_{i}(s) & =-\left(\Lambda^{i}(s)-B\right)
\end{aligned}
$$

and using Frullani's integral, we find that

$$
\begin{aligned}
I^{G}(s, z) & =\int_{0}^{+\infty}\left[\frac{e^{-\alpha_{i}(s, z) x}-e^{-\beta_{i}(s) x}}{x}-z \frac{e^{-\gamma_{i}(s) x}-e^{-\beta_{i}(s) x}}{x}\right] d x \\
& =\log \left(\frac{\beta_{i}(s)}{\alpha_{i}(s, z)}\right)-z \log \left(\frac{\beta_{i}(s)}{\gamma_{i}(s)}\right) \\
& =\log \left(\frac{\Lambda^{i-1}(s)-B}{z \lambda\left(s, T_{i}^{*}\right)+\Lambda^{i-1}(s)-B}\right)-z \log \left(\frac{\Lambda^{i-1}(s)-B}{\Lambda^{i}(s)-B}\right) .
\end{aligned}
$$

Therefore, we get the form

$$
\begin{aligned}
M_{X_{T_{i}^{*}}}(z)= & \left(F\left(0, T_{i}^{*}, T_{i-1}^{*}\right)\right)^{z} \exp \left(\int_{0}^{T_{i}^{*}} \frac{c_{s} z}{2}(z-1) \lambda^{2}\left(s, T_{i}^{*}\right) d s\right) \\
& \times \exp \left(\int_{0}^{T_{i}^{*}} \dot{A}(s) \log \left(\frac{\Lambda^{i-1}(s)-B}{z \lambda\left(s, T_{i}^{*}\right)+\Lambda^{i-1}(s)-B}\right) d s\right) \\
& \times \exp \left(-z \int_{0}^{T_{i}^{*}} \dot{A}(s) \log \left(\frac{\Lambda^{i-1}(s)-B}{\Lambda^{i}(s)-B}\right) d s\right)
\end{aligned}
$$

or

$$
\begin{aligned}
M_{X_{T_{i}^{*}}}(R+\mathrm{i} u)= & \left(F\left(0, T_{i}^{*}, T_{i-1}^{*}\right)\right)^{R+\mathrm{i} u} \\
& \times \exp \left(\int_{0}^{T_{i}^{*}} \frac{c_{s}}{2}(R+\mathrm{i} u)(R+\mathrm{i} u-1) \lambda^{2}\left(s, T_{i}^{*}\right) d s\right) \\
& \times \exp \left(\int_{0}^{T_{i}^{*}} \dot{A}(s) \log \left(\frac{\Lambda^{i-1}(s)-B}{(R+\mathrm{i} u) \lambda\left(s, T_{i}^{*}\right)+\Lambda^{i-1}(s)-B}\right) d s\right) \\
& \times \exp \left(-(R+\mathrm{i} u) \int_{0}^{T_{i}^{*}} \dot{A}(s) \log \left(\frac{\Lambda^{i-1}(s)-B}{\Lambda^{i}(s)-B}\right) d s\right) .
\end{aligned}
$$

The valuation formula becomes

$$
\begin{aligned}
\operatorname{Cplt}_{0}\left(T_{i}^{*}, K\right) & =\frac{B\left(0, T_{i-1}^{*}\right)}{2 \pi} \int_{\mathbb{R}} \frac{\widetilde{K}_{i}^{1-R-\mathrm{i} u} M_{X_{T_{i}^{*}}}(R+\mathrm{i} u)}{(R+\mathrm{i} u)(R+\mathrm{i} u-1)} d u \\
& =\frac{\widetilde{K}_{i} B\left(0, T_{i-1}^{*}\right)}{2 \pi} \int_{\mathbb{R}} \frac{d u}{(R+\mathrm{i} u)(R+\mathrm{i} u-1)}\left\{\left(\frac{F\left(0, T_{i}^{*}, T_{i-1}^{*}\right)}{\widetilde{K}_{i}}\right)^{R+\mathrm{i} u}\right.
\end{aligned}
$$




$$
\begin{aligned}
& \times \exp \left(\int_{0}^{T_{i}^{*}} \frac{c_{s}}{2}(R+\mathrm{i} u)(R+\mathrm{i} u-1) \lambda^{2}\left(s, T_{i}^{*}\right) d s\right) \\
& \times \exp \left(\int_{0}^{T_{i}^{*}} \dot{A}(s) \log \left(\frac{\Lambda^{i-1}(s)-B}{(R+\mathrm{i} u) \lambda\left(s, T_{i}^{*}\right)+\Lambda^{i-1}(s)-B}\right) d s\right) \\
& \left.\times \exp \left(-\int_{0}^{T_{i}^{*}}(R+\mathrm{i} u) \dot{A}(s) \log \left(\frac{\Lambda^{i-1}(s)-B}{\Lambda^{i}(s)-B}\right) d s\right)\right\} .
\end{aligned}
$$

The Greek Delta is given by

$$
\begin{aligned}
\Delta\left(F\left(0, T_{i}^{*}, T_{i-1}^{*}\right)\right)= & \frac{B\left(0, T_{i-1}^{*}\right)}{2 \pi} \int_{\mathbb{R}}\left\{\left(\frac{F\left(0, T_{i}^{*}, T_{i-1}^{*}\right)}{\widetilde{K}_{i}}\right)^{R+\mathrm{i} u-1}\right. \\
& \times \exp \left(\int_{0}^{T_{i}^{*}} \frac{c_{s}}{2}(R+\mathrm{i} u)(R+\mathrm{i} u-1) \lambda^{2}\left(s, T_{i}^{*}\right) d s\right) \\
& \times \exp \left(\int_{0}^{T_{i}^{*}} \dot{A}(s) \log \left(\frac{\Lambda^{i-1}(s)-B}{(R+\mathrm{i} u) \lambda\left(s, T_{i}^{*}\right)+\Lambda^{i-1}(s)-B}\right) d s\right) \\
& \left.\quad \times \exp \left(-\int_{0}^{T_{i}^{*}}(R+\mathrm{i} u) \dot{A}(s) \log \left(\frac{\Lambda^{i-1}(s)-B}{\Lambda^{i}(s)-B}\right) d s\right)\right\} \frac{d u}{R+\mathrm{i} u-1} .
\end{aligned}
$$

Acknowledgments The KPMG Center of Excellence in Risk Management is acknowledged for organizing the conference "Challenges in Derivatives Markets - Fixed Income Modeling, Valuation Adjustments, Risk Management, and Regulation”.

\section{A Appendix}

\section{A.1 Isonormal Lévy Process (ILP)}

Let $\mu$ and $v$ be $\sigma$-finite measures without atoms on the measurable spaces (T, $\mathscr{A}$ ) and $\left(\mathrm{T} \times \mathrm{X}_{0}, \mathscr{B}\right)$, respectively. Define a new measure

$$
\pi(d t, d z):=\mu(d t) \delta_{\Theta}(d z)+v(d t, d z)
$$

on a measurable space $(\mathrm{T} \times \mathrm{X}, \mathscr{G})$, where $\mathrm{X}=\mathrm{X}_{0} \cup\{\Theta\}, \mathscr{G}=\sigma(\mathscr{A} \times\{\Theta\}, \mathscr{B})$ and $\delta_{\Theta}(d z)$ is the measure which gives mass one to the point $\Theta$. We assume that the Hilbert space $\mathrm{H}:=\mathrm{L}^{2}(\mathrm{~T} \times \mathrm{X}, \mathscr{G}, \pi)$ is separable.

Definition A.1 We say that a stochastic process $\mathrm{L}=\{\mathrm{L}(h), h \in \mathrm{H}\}$ defined on a complete probability space $(\Omega, \mathscr{F}, P)$ is an isonormal Lévy process (or Lévy process on $H$ ) if the following conditions are satisfied:

1. The mapping $h \longrightarrow L(h)$ is linear; 
2. $\mathrm{E}\left[e^{i x L(h)}\right]=\exp (\Psi(x, h))$, where $\Psi(x, h)$ is equal to

$$
\int_{\mathrm{T} \times \mathrm{X}}\left(\left(e^{\mathrm{i} x h(t, z)}-1-\mathrm{i} x h(t, z)\right) \mathbf{1}_{\mathrm{X}_{0}}(z)-\frac{1}{2} x^{2} h^{2}(t, z) \mathbf{1}_{\Theta}(z)\right) \pi(d t, d z) .
$$

\section{A.2 The Derivative Operator}

Let $\mathscr{S}$ denote the class of smooth random variables, that is the class of random variables $\xi$ of the form

$$
\xi=f\left(L\left(h_{1}\right), \ldots, L\left(h_{n}\right)\right),
$$

where $f$ belongs to $\mathrm{C}_{b}^{\infty}\left(\mathbb{R}^{n}\right), h_{1}, \ldots, h_{n}$ are in $H$, and $n \geq 1$. The set $\mathscr{S}$ is dense in $L^{p}(\Omega)$ for any $p \geq 1$.

Definition A.2 The stochastic derivative of a smooth random variable of the form (100) is the $H$-valued random variable $D \xi=\left\{D_{t, x} \xi,(t, x) \in T \times X\right\}$ given by

$$
\begin{aligned}
D_{t, x} \xi= & \sum_{k=1}^{n} \frac{\partial f}{\partial y_{k}}\left(L\left(h_{1}\right), \ldots, L\left(h_{n}\right)\right) h_{k}(t, x) \mathbf{1}_{\Theta}(x) \\
& +\left(f\left(L\left(h_{1}\right)+h_{1}(t, x), \ldots, L\left(h_{n}\right)+h_{n}(t, x)\right)\right. \\
& \left.-f\left(L\left(h_{1}\right), \ldots, L\left(h_{n}\right)\right)\right) \mathbf{1}_{X_{0}}(x) .
\end{aligned}
$$

We will consider $D \xi$ as an element of $L^{2}(T \times X \times \Omega) \cong L^{2}(\Omega ; H)$; namely $D \xi$ is a random process indexed by the parameter space $T \times X$.

1. If the measure $v$ is zero or $h_{k}(t, x)=0, k=1, \ldots, n$ when $x \neq \Theta$ then $D \xi$ coincides with the Malliavin derivative (see, e.g. Nualart (2006) [15] Definition 1.2.1 p.38).

2. If the measure $\mu$ is zero or $h_{k}(t, x)=0, k=1, \ldots, n$ when $x=\Theta$ then $D \xi$ coincides with the difference operator (see, e.g. Picard (1996) [18]).

\section{A.3 Integration by Parts Formula}

Theorem A.3 Suppose that $\xi$ and $\eta$ are smooth random variables and $h \in H$. Then 1 .

$$
\mathrm{E}[\xi L(h)]=\mathrm{E}\left[\langle D \xi ; h\rangle_{H}\right]
$$


2.

$$
\mathrm{E}[\xi \eta L(h)]=\mathrm{E}\left[\eta\langle D \xi ; h\rangle_{H}\right]+\mathrm{E}\left[\xi\langle D \eta ; h\rangle_{H}\right]+\mathrm{E}\left[\left\langle D \eta ; h \mathbf{1}_{X_{0}} D \xi\right\rangle_{H}\right]
$$

As a consequence of the above theorem we obtain the following result:

The expression of the derivative $D \xi$ given in (101) does not depend on the particular representation of $\xi$ in (100).

The operator $D$ is closable as an operator from $L^{2}(\Omega)$ to $L^{2}(\Omega ; H)$.

We will denote the closure of $D$ again by $D$ and its domain in $L^{2}(\Omega)$ by $\mathbb{D}^{1,2}$.

\section{A.4 The Chain Rule}

Proposition A.4 (see Yablonski (2008), Proposition 4.8) Suppose $F=\left(F_{1}, F_{2}\right.$, $\left.\ldots, F_{n}\right)$ is a random vector whose components belong to the space $\mathbb{D}^{1,2}$. Let $\phi \in$ $\mathscr{C}^{1}\left(\mathbb{R}^{n}\right)$ be a function with bounded partial derivatives such that $\phi(F) \in \mathrm{L}^{2}(\Omega)$. Then $\phi(F) \in \mathbb{D}^{1,2}$ and

$$
D_{t, x} \phi(F)=\left\{\begin{array}{lr}
\sum_{i=1}^{n} \frac{\partial \phi}{\partial x_{i}}(F) D_{t, \Theta} F_{i} ; & x=\Theta \\
\phi\left(F_{1}+D_{t, x} F_{1}, \ldots, F_{n}+D_{t, x} F_{n}\right)-\phi\left(F_{1}, \ldots, F_{n}\right) ; & x \neq \Theta .
\end{array}\right.
$$

\section{A.5 Regularity of Solutions of SDEs Driven by Time-Inhomogeneous Lévy Processes}

We focus on a class of models in which the price of the underlying asset is given by the following stochastic differential equation (see Di Nunno et al. [2] and Petrou [17] for details)

$$
\begin{aligned}
d S_{t}= & b\left(t, S_{t-}\right) d t+\sigma\left(t, S_{t-}\right) d W_{t} \\
& +\int_{\mathbb{R}_{0}} \varphi\left(t, S_{t-}, z\right) \tilde{N}(d t, d z), \\
S_{0}= & x
\end{aligned}
$$

where $\mathbb{R}_{0}:=\mathbb{R}^{d} \backslash\left\{0_{\mathbb{R}^{d}}\right\}, x \in \mathbb{R}^{d},\left\{W_{t}, 0 \leq t \leq T\right\}$ is an $m$-dimensional standard Brownian motion, $\tilde{N}$ is a compensated Poisson random measure on $[0, T] \times$ $\mathbb{R}_{0}$ with compensator $v_{t}(d z) d t$. The coefficients $b: \mathbb{R}^{+} \times \mathbb{R}^{d} \longrightarrow \mathbb{R}^{d}, \sigma: \mathbb{R}^{+} \times$ $\mathbb{R}^{d} \longrightarrow \mathbb{R}^{d} \times \mathbb{R}^{m}$ and $\varphi: \mathbb{R}^{+} \times \mathbb{R}^{d} \times \mathbb{R} \longrightarrow \mathbb{R}^{d} \times \mathbb{R}$, are continuously differentiable with bounded derivatives and the family of positive measures $\left(v_{t}\right)_{t \in[0, T]}$ satisfies $\int_{0}^{T}\left(\int_{\mathbb{R}_{0}}\left(\|z\|^{2} \wedge 1\right) v_{t}(d z)\right) d t<\infty$ and $v_{t}(\{0\})=0$. The coefficients are assumed to satisfy the following linear growth condition 


$$
\|b(t, x)\|^{2}+\|\sigma(t, x)\|^{2}+\int_{\mathbb{R}_{0}}\|\varphi(t, x, z)\|^{2} v_{t}(d z) \leq C\left(1+\|x\|^{2}\right),
$$

for all $t \in[0, T], x \in \mathbb{R}^{d}$, where $C$ is a positive constant. Furthermore, we suppose that there exists a function $\rho: \mathbb{R} \longrightarrow \mathbb{R}$ with

$$
\sup _{0 \leq t \leq T} \int_{\mathbb{R}_{0}}|\rho(z)|^{2} v_{t}(d z)<\infty,
$$

and a positive constant $K$ such that

$$
\|\varphi(t, x, z)-\varphi(t, y, z)\| \leq K|\rho(z)|\|x-y\|,
$$

for all $t \in[0, T], x, y \in \mathbb{R}^{d}$ and $z \in \mathbb{R}_{0}$.

In the sequel we provide a theorem which proves that under specific conditions the solution of a stochastic differential equation belongs to the domain $\mathbb{D}^{1,2}$.

Theorem A.5 Let $\left(S_{t}\right)_{t \in[0, T]}$ be the solution of (105). Then $S_{t} \in \mathbb{D}^{1,2}$ for all $t \in$ $[0, T]$ and the derivative $D_{r, 0} S_{t}$ satisfies the following linear equation

$$
\begin{aligned}
D_{r, 0} S_{t}= & \int_{r}^{t} \frac{\partial b}{\partial x}\left(u, S_{u-}\right) D_{r, 0} S_{u-} d u+\sigma\left(r, S_{r-}\right) \\
& +\int_{r}^{t} \frac{\partial \sigma}{\partial x}\left(u, S_{u-}\right) D_{r, 0} S_{u-} d W_{u} \\
& +\int_{r}^{t} \int_{\mathbb{R}_{0}} \frac{\partial \varphi}{\partial x}\left(u, S_{u-}, y\right) D_{r, 0} S_{u-} \tilde{N}(d u, d y)
\end{aligned}
$$

for $0 \leq r \leq t$ a.e. and $D_{r, 0} S_{t}=0$ a.e. otherwise.

As in the classical Malliavin calculus we are able to associate the solution of (105) to the first variation process $Y_{t}:=\nabla_{x} S_{t}$. Then, we will also provide a specific expression for $D_{r, 0} S_{t}$, the Wiener directional derivative of the $S_{t}$.

Proposition A.6 Let $\left(S_{t}\right)_{t \in[0, T]}$ be the solution of (105). Then the derivative satisfies the following equation

$$
D_{r, 0} S_{t}=Y_{t} Y_{r-}^{-1} \sigma\left(r, S_{r-}\right) \mathbf{1}_{\{r \leq t\}} \text { a.e. }
$$

where $\left(Y_{t}\right)_{t \in[0, T]}$ is the first variation process of $\left(S_{t}\right)_{t \in[0, T]}$.

Open Access This chapter is distributed under the terms of the Creative Commons Attribution 4.0 International License (http://creativecommons.org/licenses/by/4.0/), which permits use, duplication, adaptation, distribution and reproduction in any medium or format, as long as you give appropriate credit to the original author(s) and the source, a link is provided to the Creative Commons license and any changes made are indicated.

The images or other third party material in this chapter are included in the work's Creative Commons license, unless indicated otherwise in the credit line; if such material is not included 
in the work's Creative Commons license and the respective action is not permitted by statutory regulation, users will need to obtain permission from the license holder to duplicate, adapt or reproduce the material.

\section{References}

1. Berman, M.: Inhomogeneous and modulated gamma processes. Biometrika 68, 143-152 (1981)

2. Di Nunno, G., Øksendal, B., Proske, F.: Malliavin Calculus for Lévy Processes with Applications to Finance. Springer, Berlin (2008)

3. Eberlein, E.: Fourier-based valuation methods in mathematical finance. In: Benth, F.E., Kholodnyi, V.A., Laurence, P. (eds.) Quantitative Energy Finance, pp. 85-114. Springer, New York (2014)

4. Eberlein, E., Keller, U.: Hyperbolic distributions in finance. Bernoulli 1(3), 281-299 (1995)

5. Eberlein, E., Özkan, F.: The Lévy LIBOR model. Financ. Stochast. 9, 327-348 (2005)

6. Eberlein, E., Kluge, W.: Exact pricing formulae for caps and swaptions in a Lévy term structure model. J. Comput. Financ. 9(2), 99-125 (2006)

7. Eberlein, E., Kluge, W.: Calibration of Lévy term structure models. In: Fu, M., Jarrow, R., Yen, J.-Y., Elliott, R. (eds.) Advances in Mathematical Finance, Applied and Numerical Harmonic Analysis, pp. 147-172. Birkhäuser, Boston (2007)

8. Eberlein, E., Glau, K., Papapantoleon, A.: Analysis of Fourier transform valuation formulas and applications. Appl. Math. Financ. 17, 211-240 (2010)

9. Fournié, E., Lasry, J.-M., Lebuchoux, J., Lions, P.-L., Touzi, N.: Applications of Malliavin calculus to Monte Carlo methods in finance. Financ. Stochast. 3(4), 391-412 (1999)

10. Fries, C.: Mathematical Finance: Theory, Modeling, Implementation. Wiley, Chichester (2007)

11. Glasserman, P.: Monte Carlo Methods in Financial Engineering. Springer, New York (2004)

12. Glasserman, P., Zhao, X.: Fast Greeks by simulation in forward LIBOR models. J. Comput. Financ. 3(1), 5-39 (1999)

13. Jacod, J., Shiryaev, A.N.: Limit Theorems for Stochastic Processes. Springer, Berlin (1987)

14. León, J.A., Solé, J.L., Utzet, F., Vives, J.: On Lévy processes, Malliavin calculus and market models with jumps. Financ. Stochast. 6, 197-225 (2002)

15. Nualart, D.: The Malliavin Calculus and Related Topics, 2nd edn. Springer, Berlin (2006)

16. Ostrowski, A.M.: On some generalizations of the Cauchy-Frullani integral. Proc. Natl. Acad. Sci. 35, 612-615 (1949)

17. Petrou, E.: Malliavin calculus in Lévy spaces and applications to finance. Electron. J. Probab. 13(27), 852-879 (2008)

18. Picard, J.: On the existence of smooth densities for jump processes. Probab. Theory Related Fields 105(4), 481-511 (1996)

19. Yablonski, A.: The calculus of variations for processes with independent increments. Rocky Mt. J. Math. 38(2), 669-701 (2008) 